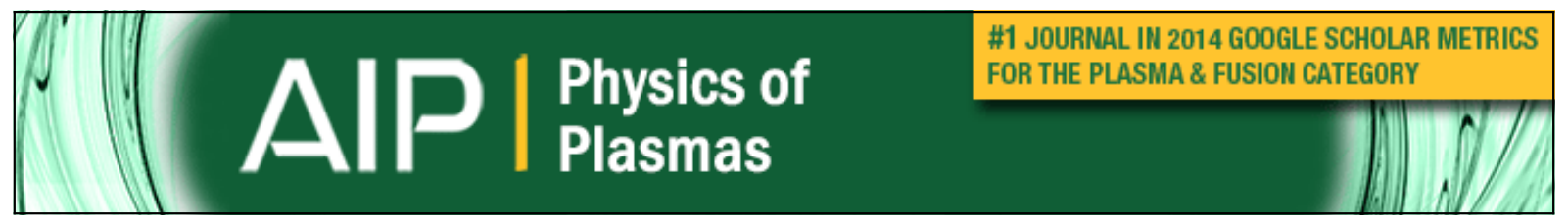

\title{
Equilibrium of non-neutral plasmas in a Malmberg-Penning trap with a weakly tilted magnetic field
}

Igor Kotelnikov and Massimiliano Romé

Citation: Physics of Plasmas 15, 072118 (2008); doi: 10.1063/1.2961074

View online: http://dx.doi.org/10.1063/1.2961074

View Table of Contents: http://scitation.aip.org/content/aip/journal/pop/15/7?ver=pdfcov

Published by the AIP Publishing

\section{Articles you may be interested in}

Effect of a Weakly Tilted Magnetic Field on the Equilibrium of Nonneutral Plasmas in a Malmberg-Penning Trap AIP Conf. Proc. 1114, 130 (2009); 10.1063/1.3122275

Equilibrium of non-neutral plasmas with weak axisymmetric magnetic perturbations

AIP Conf. Proc. 862, 116 (2006); 10.1063/1.2387915

Decay of trapped-particle asymmetry modes in non-neutral plasmas in a Malmberg-Penning trap Phys. Plasmas 10, 1231 (2003); 10.1063/1.1566959

A magnetic trap for simultaneous confinement of neutral atoms and a non-neutral plasma AIP Conf. Proc. 606, 582 (2002); 10.1063/1.1454334

Non-neutral plasma expansion induced by electron-neutral collisions in a Malmberg-Penning trap J. Vac. Sci. Technol. A 17, 2050 (1999); 10.1116/1.581724

\section{Did your publisher get}

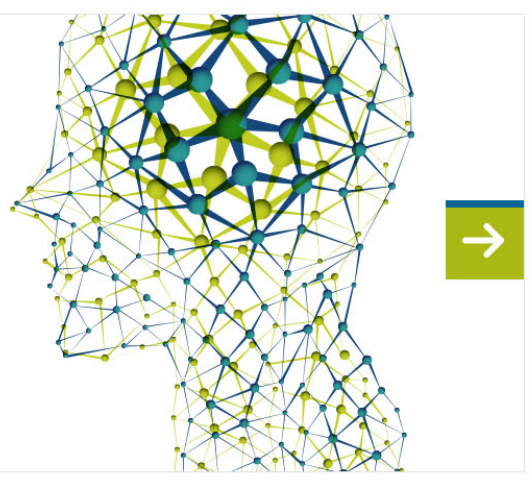




\title{
Equilibrium of non-neutral plasmas in a Malmberg-Penning trap with a weakly tilted magnetic field
}

\author{
Igor Kotelnikov ${ }^{1}$ and Massimiliano Romé ${ }^{2}$ \\ ${ }^{1}$ Budker Institute of Nuclear Physics, Lavrentyev Av. 11, Novosibirsk, 630090, Russia \\ ${ }^{2}$ I.N.F.N. Sezione di Milano and Dipartimento di Fisica, Università degli Studi di Milano, \\ Via Celoria 16, I-20133 Milano, Italy
}

(Received 15 May 2008; accepted 27 June 2008; published online 31 July 2008)

\begin{abstract}
The effect of small asymmetric magnetic perturbations on the equilibrium of a non-neutral plasma confined in a Malmberg-Penning trap is analyzed. A constraint, known in the theory of tandem mirrors as the condition of current closure, is derived for non-neutral plasmas. Together with Poisson's equation, this constraint provides a set of equations for determining self-consistent asymmetric equilibria of non-neutral plasmas in Malmberg-Penning traps. As an example of this approach, the non-neutral plasma equilibrium in the presence of a weak magnetic tilt is analyzed. Analytical and semianalytical solutions for the electric potential variations inside the trap are found in a paraxial limit for various radial density profiles of the plasma, including the case of global thermal equilibrium. The numerical procedure aimed to obtain self-consistent plasma equilibria for a magnetic field with a large asymmetry is also discussed. The newly developed method can be straightforwardly applied to determine plasma equilibria under the effect of the magnetic perturbations of higher multipolarity (such as, quadrupole or octupole fields). (C) 2008 American Institute of Physics. [DOI: 10.1063/1.2961074]
\end{abstract}

\section{INTRODUCTION}

The radial confinement of non-neutral plasmas in Malmberg-Penning traps is provided by a strong axial magnetic field. This field is assumed to be uniform in most theories that deal with plasma confinement. However it has long been suspected that small perturbations of the magnetic field may play a crucial role in the transport of non-neutral plasmas in this kind of confinement devices; ${ }^{1}$ see also the review paper 2, and references therein for further discussion of the problem of non-neutral plasma transport. On the other hand, it is well known that an accurate treatment of the plasma transport requires at first an analysis of the plasma equilibrium, as it is proven by established theories for quasineutral plasma confined, e.g., in tandem mirrors. ${ }^{3}$ This fact was neglected in some previous theories of non-neutral plasma transport induced by magnetic field errors (see, e.g., Refs. $5-8)$.

Systematic studies of nonaxisymmetric equilibria in a Malmberg-Penning trap have been started in Refs. 9 and 10. In Ref. 9 the equilibrium of a non-neutral plasma column in a weakly tilted magnetic field was simulated numerically. In Ref. 10 a static electrostatic asymmetry was introduced by azimuthally sectored electrodes, and the analytical treatment was limited to the case of a cold plasma with a stepwise radial density profile. Later on, three-dimensional (3D) numerical particle-in-cell (PIC) simulations of the non-neutral plasma equilibrium with quadrupole or mirror magnetic perturbations have been reported in Ref. 11. However, similar numerical simulations are hardly able to uncover finestructure effects that limit plasma lifetime in existing and future facilities designed to achieve improved confinement of non-neutral plasmas (relevant, e.g., to antimatter studies ${ }^{12,13}$ ).

The present paper is a natural development of a recent work by the authors, ${ }^{14}$ where the effect of small axisymmetric perturbations of the magnetic field on the equilibrium of a non-neutral plasma in a Malmberg-Penning trap was considered both analytically and numerically. The approach is based on the use of curvilinear flux coordinates for the magnetic field. As it was argued in Ref. 14, performing the calculations in flux coordinates makes the interpretation of the plasma equilibrium much easier and provides the best approach to the problem of the error field mediated transport. In particular, it was shown that if a magnetic perturbation is turned on "adiabatically" in a given section of the confinement device, then the associated perturbation of the electric potential in a given point turns out to be generally much greater then the potential perturbation on a fixed magnetic field line. In other words, a magnetic flux surface within the charged plasma column remains quasi-equipotential if the magnetic field becomes nonuniform. This makes the use of curvilinear coordinates preferable when computing the electric field in charged plasmas confined in slightly nonuniform magnetic fields. It will be shown here that similar considerations are valid for asymmetric perturbations as well.

In Ref. 15, the equilibrium of non-neutral plasmas on a set of nested toroidal magnetic surfaces has been recently considered. This work together with the theory of quasineutral plasma equilibria in tandem mirrors ${ }^{3}$ bestows a guideline of how to establish a constraint on the shape of admissible plasma equilibria. Together with Poisson's equation, rewritten in flux coordinates, this constraint constitutes a selfconsistent method for determining asymmetric equilibria of non-neutral plasmas in a Malmberg-Penning trap. This method can be applied to any kind of asymmetry, such as, quadrupole or octupole fields, but in the present paper the 
study is focused on the case of a weakly tilted magnetic field perturbation.

The paper is organized as follows: In Sec. II, the main features of the model used to analyze the effect of magnetic field perturbations are outlined, namely, the meaning of "plasma equilibrium" is explained in circumstances which cause the plasma to expand. In Sec. III curvilinear coordinates are introduced and magnetic field perturbations with different multipolarity (specified by a given value of the azimuthal mode number $m$ ) are briefly analyzed. In Sec. IV a solvability condition is derived that constraints the shape of the flux surfaces. This condition has a direct analog in the theory of quasineutral plasma equilibrium in tandem mirrors. ${ }^{3}$ In the theory of tokamaks and stellarators a similar condition is known as parallel current constraint. ${ }^{4}$

On the other hand, the effect of the image charges due to the presence of the conducting walls makes the treatment for a non-neutral plasma much more difficult than that for a neutral plasma. The parallel current constraint also distinguishes the present treatment from that in Ref. 15, where toroidal configurations of non-neutral plasmas are analyzed and the longitudinal current density remains undetermined.

In Sec. $\mathrm{V}$ the Poisson equation in curvilinear coordinates is derived, with special emphasis on the case of weak magnetic field perturbations. In Sec. VI the formalism developed in the previous sections is applied to the plasma equilibrium in a Malmberg-Penning trap with a weak magnetic squeeze, $m=0$. This case was extensively treated in Ref. 14 but it is revisited in the context of the new general approach developed here.

In Sec. VII a preliminarily analysis of a weakly tilted magnetic field, i.e., a dipole magnetic perturbation with $m$ $=1$, is performed. It is shown in particular that a magnetic tilt differs significantly from other types of magnetic perturbations since it is effectively shielded at the plasma edge. In Sec. VIII a self-consistent solution is found for the plasma equilibrium in a Malmberg-Penning trap in the case of a week uniform magnetic tilt. A second example of an analytically solvable equilibrium is given in Sec. IX for the case of a so-called "reversible magnetic tilt." In contrast to the case of a uniform magnetic tilt, this kind of perturbation penetrates into the plasma core, i.e., the electric potential close to the plasma column axis turns out to be non-negligibly perturbed.

In Sec. X longitudinal plasma currents are calculated. They can be considered as a direct analog of the PfirschSchlüter currents in tokamaks or the Stupakov currents in tandem mirrors, although they have a quite different origin. Namely, both the Pfirsch-Schlüter ${ }^{16}$ and the Stupakov ${ }^{17}$ currents originate from magnetic drifts, whereas longitudinal currents appear in a non-neutral plasma even in the case of a uniformly tilted magnetic field, i.e., in the absence of magnetic drifts of any kind.

In Sec. XI the application of the present method for the numerical computation of plasma equilibria in cases where the magnetic asymmetry may not be considered as small, is outlined. In Sec. XII the main results are summarized and possible further developments are sketched.

Appendix A presents the derivation of a formula that is required to prove the equivalence of the Poisson equation for a weakly inhomogeneous magnetic field presented in Sec. V with that previously obtained by O'Neil. ${ }^{18}$ And finally, Appendix B contains explicit formulas (valid in the particular case of a weak magnetic tilt) for the transformation from the flux coordinates to the ordinary cylindrical coordinates naturally related to the plasma confinement device.

\section{MODEL}

One may wonder whether an equilibrium of a nonneutral plasma exists in an asymmetric magnetic field, since an asymmetry leads in general to plasma expansion. A positive answer to this question implies that the equilibrium is referred to a time interval shorter than the expansion time $\tau_{m}$. If the asymmetry is small, the latter is expected to be at least greater then the axial bounce time of the particles inside the trap, $\tau_{b}$, and the plasma azimuthal rotation time, $2 \pi / \omega_{E}$, i.e., $\tau_{m} \gg\left(\tau_{b}, 2 \pi / \omega_{E}\right)$. In general, $\tau_{m} \propto \epsilon^{-2}$, where the parameter $\epsilon$ characterizes the smallness on the magnetic field inhomogeneity. At this stage it can be thought of as $\epsilon \sim \delta B / B$, where $\delta \mathbf{B}$ represents the difference of the actual magnetic field from an ideal uniform magnetic field $\mathbf{B}_{*}=B_{*} \mathbf{e}_{z}$ directed along the symmetry axis of the cylindrical confinement device. For the small $\epsilon$ values achieved in existing devices the expansion time can therefore be quite large, and for a shorter time interval, $t \ll \tau_{m}$, it is possible to consider a slowly evolving plasma column as being in a static equilibrium.

If a plasma equilibrium exists in the sense detailed above, it should be emphasized that a small transverse offset of the plasma column creates a new stationary state in which the plasma rotates around its own axis and simultaneously drifts around the device axis with the diocotron frequency, the latter being equal to the electric drift frequency at the wall of the confinement device. This new state represents a neutrally stable $m=1$ diocotron mode. There is vast literature devoted to the fundamental diocotron mode; here it is sufficient to mention only the papers most relevant to the problem under consideration like, e.g., Refs. 9 and 19-21. Whether this mode exists or not depends mainly on how the plasma column is created in the experiment. In this paper such quasiequilibria are not considered, assuming that the diocotron mode is somehow suppressed.

Some theories (see, e.g., Refs. 5 and 6) consider a collisionless plasma expansion in the presence of a static magnetic field perturbation. This collisionless expansion may be interpreted as an actual absence of equilibrium. In any case it is undoubtful that the expansion becomes slower as the asymmetry becomes smaller. Note also that the theory of resonant transport ${ }^{3}$ predicts the existence of a stochastic regime of the transverse transport of a quasineutral plasma in tandem mirrors, where the diffusion coefficient formally does not depend on the collision rate. This does not mean, however, that such expansion is really collisionless since without collisions it would be reversible (analogously, e.g., to the reversibility of the Landau damping, discovered by the existence of the phenomenon of plasma echo).

In this paper, a model of a long pure electron plasma column contained in a cylindrical conducting chamber of 

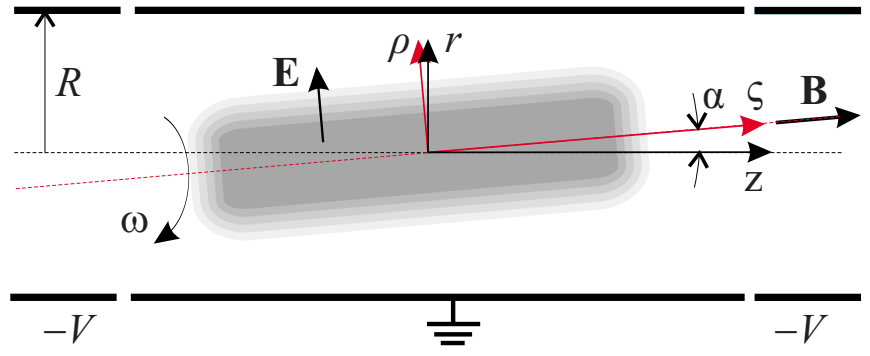

FIG. 1. (Color online) Effect of a tilt of the magnetic field on the equilibrium of a long non-neutral plasma column, confined inside an ideally conducting vacuum chamber with a circular cross section of radius $R$. The plasma is confined radially be the external magnetic field and longitudinally by electrostatic plugs. The magnetic coils are located outside of the cylindrical chamber and are not shown. Throughout the paper, the cylindrical coordinates $(r, \theta, z)$ (black arrows) naturally related to the conducting chamber are referred to as device coordinates to distinguish them from the flux coordinates $(\rho, \vartheta, \zeta)$ (red arrows), for which a magnetic field line is determined by given values of $\rho$ and $\vartheta$ (while $\zeta$ varies along the line), and the radius $a$ of the plasma column is defined in flux coordinates by the position of one half of the maximum plasma density (located at the column axis).

radius $R$ and immersed in a weakly tilted magnetic field $\mathbf{B}$ is adopted, as shown in Fig. 1. The attention is focused on the central part of the confining chamber, with a grounded conducting wall, where the electrostatic potential $\phi$ is assumed to be zero, $\phi=0$. In the unperturbed state, characterized by a uniform magnetic field $\mathbf{B}_{*}$, the plasma density is constant along field lines. The aim is to characterize the electric potential in the plasma in those regions of the device where the magnetic field $\mathbf{B}=\mathbf{B}_{*}+\delta \mathbf{B}$ is perturbed by a small quantity $\delta B \ll B_{*}$.

In the next section suitable curvilinear coordinates are introduced to describe the magnetic field. In Sec. IV they will be used for the derivation of the parallel current constraint, and the Poisson equation will be explicitly written in these curvilinear coordinates in Sec. V.

\section{MAGNETIC FIELD}

The electric current produced by the flowing electrons in a column with radius $a$ produces a negligible change of the magnetic field,

$$
\frac{\delta B}{B} \approx \frac{1}{16}\left(\frac{n}{n_{B}}\right)^{2}\left(\frac{a}{c / \omega_{c}}\right)^{2},
$$

if the electron density $n$ is far below the Brillouin limit, ${ }^{22}$ defined as

$$
n_{B}=\frac{B^{2}}{8 \pi m c^{2}} .
$$

Here $\omega_{c}=e B / m c$ is the cyclotron frequency, with $e$ and $m$ the particle charge and mass, respectively, and $c$ the speed of light.

\section{A. Curvilinear coordinates}

The condition $\delta B / B \ll 1$ is usually very well satisfied in the case of slowly rotating non-neutral plasma equilibria. ${ }^{23}$ The magnetic field can then be described by a scalar magnetic potential $\chi$ such that

$$
\mathbf{B}=\nabla \chi .
$$

Alternatively, any divergence-free field can be written as

$$
\mathbf{B}=\nabla \psi \times \nabla \vartheta
$$

where $\psi$ and $\vartheta$ are flux coordinates, ${ }^{24}$ which are constant along the magnetic field lines. The relations

$$
\psi=\psi(x, y, z), \quad \vartheta=\vartheta(x, y, z), \quad \chi=\chi(x, y, z)
$$

define a system of curvilinear coordinates.

In the following, a second set of quasicylindrical curvilinear coordinates $(\rho, \vartheta, \zeta)$ will predominantly be used, which is related to $(\psi, \vartheta, \chi)$ by the equations

$$
\psi=\frac{1}{2} B_{*} \rho^{2}, \quad \chi=B_{*} \zeta .
$$

These coordinates are assumed to become ordinary cylindrical coordinates $(r, \theta, z)$ when the magnetic field $\mathbf{B}=B_{*} \mathbf{e}_{z}$ is uniform with the strength $B_{*}$ and directed along the device axis $z$.

The inverse transformation from $\xi^{i}=(\rho, \vartheta, \zeta)$ to $x^{i}$ $=(x, y, z)$ determines a vector function $\mathbf{x}=\mathbf{x}(\rho, \vartheta, \zeta)$, which gives a magnetic field line for a fixed pair $\rho, \vartheta$.

Using the coordinates $(\rho, \vartheta, \zeta)$, Eqs. (1) and (2) become

$$
\begin{aligned}
& \mathbf{B}=B_{*} \nabla \zeta, \\
& \mathbf{B}=B_{*} \rho[\nabla \rho \times \nabla \vartheta] .
\end{aligned}
$$

The covariant components of $\mathbf{B}$,

$$
B_{i}=\left(0,0, B_{*}\right),
$$

are determined by Eq. (4), and its contravariant components,

$$
B^{i}=\left(0,0, B_{*} \rho / \sqrt{g}\right),
$$

by Eq. (5), where $g=\operatorname{det}\left\|g_{i k}\right\|$ and

$$
g_{i k}=\frac{\partial \mathbf{x}}{\partial \xi^{i}} \cdot \frac{\partial \mathbf{x}}{\partial \xi^{k}}
$$

is the metric tensor. Since $B_{i}=g_{i k} B^{k}$, it follows immediately from the comparison of Eqs. (6) and (7) that $g_{13}=g_{23}=g_{31}$ $=g_{32}=0$, i.e.,

$$
g_{i k}=\left[\begin{array}{ccc}
g_{11} & g_{12} & 0 \\
g_{21} & g_{22} & 0 \\
0 & 0 & g_{33}
\end{array}\right] \text {. }
$$

In general, the coordinates $\rho$ and $\vartheta$ are not assumed to be orthogonal and, hence, $g_{12}=g_{21}$ may not be equal to zero. One can also easily prove the following relations:

$$
\begin{aligned}
& g_{33}=\sqrt{g} / \rho, \\
& g_{11} g_{22}-g_{12} g_{21}=\sqrt{g} \rho, \\
& |\mathbf{B}|^{2}=B_{*}^{2} \rho / \sqrt{g},
\end{aligned}
$$

which will be used below.

For the sake of brevity, the ordinary cylindrical coordinates $(r, \theta, z)$ will henceforth be referred to as device coor- 
dinates to distinguish them from the curvilinear coordinates $(\rho, \vartheta, \zeta)$ indicated as flux coordinates (see Fig. 1).

\section{B. Long-thin expansion}

The scalar potential $\zeta$ of the magnetic field obeys the Laplace equation,

$$
\frac{1}{r} \frac{\partial}{\partial r} r \frac{\partial \zeta}{\partial r}+\frac{1}{r^{2}} \frac{\partial^{2} \zeta}{\partial \theta^{2}}+\frac{\partial^{2} \zeta}{\partial z^{2}}=0
$$

and can be expanded into a Fourier series over the azimuthal angle $\theta$ of the cylindrical system of coordinates $(r, \theta, z)$,

$$
\zeta(r, \theta, z)=\operatorname{Re} \sum_{m=0}^{\infty} \zeta_{(m)}(r, z) e^{i m \theta} .
$$

For any given azimuthal mode number $m$ the functions $\zeta_{(m)}(r, z)$ can be written as a power series over the radius $r$,

$$
\zeta_{(m)}(r, z)=\sum_{n=0}^{\infty} \frac{(-1)^{n} m ! r^{2 n+m}}{2^{2 n} n !(m+n) !} \zeta_{(m, 0)}^{(2 n)}(z) .
$$

Here $\zeta_{(m, 0)}(z)$ is assumed to be a given function of $z$, and the superscript $(2 n)$ stands for the $2 n$th derivative over $z$. For a system with a straight (or almost straight) magnetic axis, such as the Malmberg-Penning trap, the power series (15) can be effectively truncated at the second or, at most, the third term. This truncation is known as long-thin or "paraxial" approximation. It is valid provided that the characteristic axial length of variation of the magnetic field, $L$, is much longer than its radial scale length; in the case under consideration, the latter can be considered as the radius $R$ of the conducting cylinder surrounding the plasma column.

The functions $\zeta_{(m, 0)}(z)$ may in general be complex but only the real part of $\zeta_{(m)}(r, \theta, z) e^{i m \theta}$ has a physical meaning. In order to avoid unnecessarily cumbersome expressions, it is assumed below that all functions $\zeta_{(m, 0)}(z)$ are purely real, so that terms proportional to $\sin (m \theta)$ are dropped from the following equations but they can be trivially restored if needed.

The axisymmetric part of Eq. (14) is characterized by $m=0$, and the function $\zeta_{(0,0)}$ can be conveniently expressed through the magnetic field $B_{0}(z)$ on the axis of the system, so that

$$
B_{*} \zeta_{(0)}(r, z)=\int_{z_{0}}^{z} B_{0}(z) d z-\frac{r^{2}}{4} B_{0}^{\prime}(z)+\cdots,
$$

where the prime denotes the derivative over $z$.

A dipole perturbation, $m=1$, is usually characterized by a component of the magnetic field perpendicular to the axis $z$. Since all terms proportional to $\sin (m \theta)$ are dropped, the dipole perturbation is assumed to have merely the $x$-component $B_{1 x}(z)=\operatorname{Re} \zeta_{(1,0)}$ on the axis $z$, and thus

$$
B_{*} \zeta_{(1)}(r, \theta, z) \simeq\left[B_{1 x}(z)-\frac{r^{2}}{8} B_{1 x}^{\prime \prime}(z)+\cdots\right] r \cos \theta .
$$

A quadrupole perturbation, $m=2$, is characterized by the gradient $h(z)=\operatorname{Re} \zeta_{(2,0)}$ of a transverse component of the magnetic field on the axis $z$,

$$
B_{*} \zeta_{(2)}(r, \theta, z) \simeq\left[h(z)-\frac{r^{2}}{12} h^{\prime \prime}(z)+\cdots\right] r^{2} \cos 2 \theta .
$$

Higher order perturbations can be treated analogously. For example, a sextupole perturbation, $m=3$, has the form

$$
B_{*} \zeta_{(3)}(r, \theta, z) \simeq\left[q(z)-\frac{r^{2}}{16} q^{\prime \prime}(z)+\cdots\right] r^{3} \cos 3 \theta,
$$

and an octupole perturbation, $m=4$, reads

$$
B_{*} \zeta_{(4)}(r, \theta, z) \simeq\left[u(z)-\frac{r^{2}}{20} u^{\prime \prime}(z)+\cdots\right] r^{4} \cos 4 \theta .
$$

It is shown below that the dipole perturbation (17), sometimes referred to as "magnetic tilt," represents a notable exception from the above list of magnetic perturbations of various multipolarity. In contrast to all other perturbations, including the "magnetic squeeze," $m=0$, the dipole perturbation is effectively shielded by the plasma column edge. The ability of a magnetic tilt to penetrate into the plasma column interior is comparable to that of the sextupole perturbation (19). A dipole perturbation should therefore be considered together with a sextupole perturbation, since the second term in Eq. (17) has generally the same order of magnitude of the leading term in Eq. (19). For example, taking $q=-B_{1 x}^{\prime \prime} / 24$ in Eq. (19), the sum of Eqs. (17) and (19) is

$$
B_{*} \zeta_{(1)}+B_{*} \zeta_{(2)} \simeq x B_{1 x}(z)-\frac{x^{3}}{6} B_{1 x}^{\prime \prime}(z)+\cdots .
$$

This expression can be interpreted as a "planar magnetic tilt." This kind of perturbation can be obtained using a magnetic coil with a wire winding having a rectangular cross section and slightly bent around the direction of the longer dimension. In what follows only magnetic coils with circular cross sections are considered, and therefore it is assumed that sextupole perturbations are completely absent, i.e., $q(z)=0$ everywhere.

\section{Weak inhomogeneity}

Let the formal parameter $\epsilon$ indicate the amplitude of the inhomogeneous perturbation of an almost uniform magnetic field, so that

$$
\rho=r-\epsilon \rho_{1}, \quad \vartheta=\theta-\epsilon \vartheta_{1}, \quad \zeta=z-\epsilon \zeta_{1},
$$

where $\rho_{1}, \vartheta_{1}$, and $\zeta_{1}$ are interpreted at this stage as some functions of $r, \theta$ and $z$. Eliminating in Eq. (5) all terms of order higher than $\epsilon^{1}$ yields

$$
\mathbf{B}=B_{*}\left\{\epsilon \frac{\partial \rho_{1}}{\partial z}, \epsilon r \frac{\partial \vartheta_{1}}{\partial z}, 1-\epsilon \frac{1}{r} \frac{\partial}{\partial r} r \rho_{1}-\epsilon \frac{\partial \vartheta_{1}}{\partial \theta}\right\} .
$$

The alternative form (4) of the magnetic field leads to

$$
\mathbf{B}=B_{*}\left\{-\epsilon \frac{\partial \zeta_{1}}{\partial r},-\epsilon \frac{1}{r} \frac{\partial \zeta_{1}}{\partial \theta}, 1-\epsilon \frac{\partial \zeta_{1}}{\partial z}\right\} .
$$

Equating the two expressions for the magnetic field, $\rho_{1}$ and $\vartheta_{1}$ can be written in terms of $\zeta_{1}$ as 


$$
\rho_{1}=-\int \frac{\partial \zeta_{1}}{\partial r} d z, \quad \vartheta_{1}=-\frac{1}{r^{2}} \int \frac{\partial \zeta_{1}}{\partial \theta} d z
$$

Within the adopted accuracy of order $\epsilon^{1}$ it is possible to consider $\rho_{1}, \vartheta_{1}$, and $\zeta_{1}$ in the above relations as functions of the flux coordinates $\rho, \vartheta$, and $\zeta$. Hence, one can write

$$
\rho_{1}=-\int \frac{\partial \zeta_{1}}{\partial \rho} d \zeta, \quad \vartheta_{1}=-\frac{1}{\rho^{2}} \int \frac{\partial \zeta_{1}}{\partial \vartheta} d \zeta
$$

where the function $\zeta_{1}$ obeys the Laplace equation,

$$
\frac{1}{\rho} \frac{\partial}{\partial \rho} \rho \frac{\partial \zeta_{1}}{\partial \rho}+\frac{1}{\rho^{2}} \frac{\partial^{2} \zeta_{1}}{\partial \vartheta^{2}}+\frac{\partial^{2} \zeta_{1}}{\partial \zeta^{2}}=0 .
$$

Up to the different notations, this equation coincides with Eq. (13), so that $\zeta_{1}(\rho, \vartheta, \zeta)$ is in fact already known. One needs only to take into account that the sign of $\zeta_{1}$ is opposite to that of $\zeta_{(m)}$, and that the case $m=0$ must be treated more carefully,

$$
\zeta_{1}(\rho, \vartheta, \zeta)=\zeta \delta_{0 m}-\zeta_{(m)}(\rho, \vartheta, \zeta)
$$

where the Kronecker delta $\delta_{0 m}$ is 1 if $m=0$ and 0 otherwise. Note that $\rho_{1}$ and $\vartheta_{1}$ are determined up to arbitrary functions $\rho_{10}$ and $\vartheta_{10}$ of $\rho$ and $\vartheta$. Thus, the position (22) actually implies that

$\rho_{1}=-\int_{\zeta_{0}}^{\zeta} \frac{\partial \zeta_{1}}{\partial \rho} d \zeta+\rho_{10}, \quad \vartheta_{1}=-\frac{1}{\rho^{2}} \int_{\zeta_{0}}^{\zeta} \frac{\partial \zeta_{1}}{\partial \vartheta} d \zeta+\vartheta_{10}$,

where $\zeta_{0}$ is a free parameter to be chosen below (see Sec. VIII), and the functions $\rho_{10}(\rho, \vartheta)$ and $\vartheta_{10}(\rho, \vartheta)$ are related by the equation

$$
\frac{1}{\rho} \frac{\partial}{\partial \rho} \rho \rho_{10}+\frac{\partial}{\partial \vartheta} \vartheta_{10}=0
$$

The freedom in the choice of $\rho_{10}$ and $\vartheta_{10}$ will be repeatedly used below without further notice.

In the frame of a linear approximation over the small parameter $\epsilon$, the magnetic field perturbations of various multipolarity $m$ can be treated separately. The combined effect on the plasma equilibrium of two or more magnetic perturbations is then obtained by simply summing the individual contributions.

The covariant components of the metric tensor $g_{i k}$ can now be calculated by Eq. (8). Keeping again only terms at most linear in the parameter $\epsilon$, one obtains

$$
\begin{aligned}
& g_{11}=1+2 \epsilon \frac{\partial \rho_{1}}{\partial \rho}, \quad g_{12}=\epsilon \frac{\partial \rho_{1}}{\partial \vartheta}+\epsilon \rho^{2} \frac{\partial \vartheta_{1}}{\partial \rho}, \\
& g_{22}=\left(1+2 \epsilon \frac{\rho_{1}}{\rho}+2 \epsilon \frac{\partial \vartheta_{1}}{\partial \vartheta}\right) \rho^{2}, \quad g_{33}=1+2 \epsilon \frac{\partial \zeta_{1}}{\partial \zeta} .
\end{aligned}
$$

With the same accuracy

$$
\sqrt{g}=\left(1+2 \epsilon \frac{\partial \zeta_{1}}{\partial \zeta}\right) \rho
$$

\section{ADMISSIBLE EQUILIBRIA IN A MALMBERG-PENNING TRAP}

\section{A. General properties of non-neutral plasma equilibria}

The momentum balance equation for a pure electron plasma is

$$
m n\left(\frac{\partial \mathbf{v}}{\partial t}+\mathbf{v} \cdot \nabla \mathbf{v}\right)=e n\left(\frac{1}{c} \mathbf{v} \times \mathbf{B}-\nabla \phi\right)-\nabla p,
$$

where $\mathbf{v}$ is the fluid velocity and $p$ is the scalar pressure. In the equilibrium state, the time derivative vanishes. If the electron density is far below the Brillouin limit, $n \ll n_{B}$, and the plasma column is the slow rotation state, ${ }^{23}$ then the $\mathbf{v} \cdot \nabla \mathbf{v}$ term is negligible in comparison with the other terms and the force balance equation reduces to

$$
\nabla p=e n\left(\frac{1}{c} \mathbf{v} \times \mathbf{B}-\nabla \phi\right) .
$$

Dotting $\mathbf{B}$ with Eq. (29), one finds that $\mathbf{B} \cdot \nabla p=-e n \mathbf{B} \cdot \nabla \phi$. The electron temperature $T$ tends to be constant along the magnetic field, $\mathbf{B} \cdot \nabla T=0$. When this situation is reached, the electron density must have the form

$$
n=N(\rho, \vartheta) \exp \left[-\frac{e \phi}{T(\rho, \vartheta)}\right]
$$

and must also be consistent with the Poisson equation. Therefore, the fundamental equilibrium equation for a pure electron plasma is

$$
\nabla^{2} \phi(\rho, \vartheta, \zeta)=-4 \pi e N(\rho, \vartheta) \exp \left[-\frac{e \phi}{T(\rho, \vartheta)}\right]
$$

The equation contains two functions of $\rho$ and $\vartheta, N(\rho, \vartheta)$ and $T(\rho, \vartheta)$. These functions are subject to a constraint which is derived later in this section. The dependence of $N$ and $T$ on $\vartheta$ makes the plasma equilibria in Malmberg-Penning traps very different from those obtained in toroidal devices, ${ }^{15}$ where the functions $N(\rho)$ and $T(\rho)$ are entirely determined by the experimental conditions and by the plasma transport processes.

The equilibrium equation, Eq. (29), implies

$$
\mathbf{v}=\frac{v_{\|}}{B} \mathbf{B}-c\left(\frac{\nabla p}{e n}+\nabla \phi\right) \times \frac{\mathbf{B}}{B^{2}},
$$

where $v_{\|}$denotes the component of the velocity parallel to the magnetic field. According to Eq. (30), the pressure

$$
p(\rho, \vartheta, \phi)=T(\rho, \vartheta) N(\rho, \vartheta) \exp [-e \phi / T(\rho, \vartheta)]
$$

is a function of $\rho, \vartheta$, and $\phi$. Thus,

$$
\nabla p=\frac{\partial p}{\partial \rho} \nabla \rho+\frac{\partial p}{\partial \vartheta} \nabla \vartheta-e n \nabla \phi
$$




$$
\mathbf{v}=v_{\|} \frac{\mathbf{B}}{B}-c \frac{\partial p}{\partial \rho} \frac{\nabla \rho \times \mathbf{B}}{e n B^{2}}-c \frac{\partial p}{\partial \vartheta} \frac{\nabla \vartheta \times \mathbf{B}}{e n B^{2}} .
$$

This relation reveals that $\partial p / \partial \rho$ and $\partial p / \partial \vartheta$ cannot be neglected even when $p$ vanishes in the zero temperature limit, as explained below.

The ratio of the pressure to the electrostatic term in Eq. (29) is $\left(\lambda_{D} / a\right)^{2}$, where

$$
\lambda_{D}=\sqrt{T /\left(4 \pi e^{2} n\right)}
$$

is the Debye length, and $a$ is the radius of the plasma column. The limit of small Debye length means that the plasma is cold and has negligible pressure since $|T / e \phi| \approx\left(\lambda_{D} / a\right)^{2}$. Thus the equilibrium equation for a cold pure electron plasma, $\lambda_{D} / a \rightarrow 0$, is simply

$$
\text { en } \nabla \phi=\frac{e}{c} n \mathbf{v} \times \mathbf{B} \text {. }
$$

This implies that $\mathbf{B} \cdot \nabla \phi=0$, so that the electric potential is constant along each magnetic field line. However this is apparently not true at the ends of the plasma column. It is therefore important to retain the pressure gradient in the force balance as it is done in Eq. (35).

It is remarkable that $\partial p / \partial \rho$ and sometimes $\partial p / \partial \vartheta$ do not vanish when $p$ vanishes in the zero temperature limit. A simple application of Eq. (34) gives $(\partial p / \partial \rho) \nabla \rho$ $+(\partial p / \partial \vartheta) \nabla \vartheta=n e \nabla \phi$ as $\nabla p$ vanishes. Thus, Eq. (35) reduces to

$$
\mathbf{v}=\frac{v_{\|}}{B} \mathbf{B}-c \frac{\nabla \phi \times \mathbf{B}}{B^{2}},
$$

which clearly follows from Eq. (36). Ultimately, this apparent paradox comes from the fact that the pressure depends exponentially on the factor $e \phi / T \sim\left(a / \lambda_{D}\right)^{2}$ which goes to infinity in the zero pressure limit. In the cold plasma case, even though the electrostatic potential is constant along the field lines the electron density is not, since it has to satisfy Poisson's equation (31), which in a nontrivial geometry contains the dependence on all three coordinates. In a certain sense, to evaluate the density variation along a magnetic field line in the cold plasma limit one has to take into account the presence of a finite plasma temperature, no matter how low it is.

\section{B. Parallel current constraint}

The parallel component $v_{\|}$of the plasma flow, Eq. (35), must be consistent with the steady-state constraint

$$
\nabla \cdot(e n \mathbf{v})=0 .
$$

This constraint leaves a net parallel electric current of the non-neutral plasma undetermined in a toroidal confinement configuration, as it is explained in Ref. 15, but it leads to a parallel current constraint in the case of a MalmbergPenning trap geometry as it is shown below.

Combining Eq. (35) with Eq. (38), and switching temporarily to the primary set of coordinates $(\psi, \vartheta, \chi){ }^{25}$ yields

$$
\begin{aligned}
\nabla \cdot\left(\frac{e n v_{\|}}{c B} \mathbf{B}\right)= & \nabla\left[\frac{1}{B^{2}} \frac{\partial p}{\partial \psi}\right] \cdot \nabla \psi \times \nabla \chi \\
& +\nabla\left[\frac{1}{B^{2}} \frac{\partial p}{\partial \vartheta}\right] \cdot \nabla \vartheta \times \nabla \chi
\end{aligned}
$$

The left-hand side is transformed according to

$$
\nabla \cdot\left(\frac{e n v_{\|}}{c B} \mathbf{B}\right)=B^{2} \frac{\partial}{\partial \chi} \frac{e n v_{\|}}{c B} .
$$

When calculating the gradients in the right-hand side, one needs to take into account that triple products with two gradients of the same function are equal to zero, for example, $\nabla \psi \nabla \psi \times \nabla \chi=0$. Reminded that $B$ is considered here as a function of $\psi, \vartheta$ and $\chi$, and $p$ is a function of $\psi, \vartheta$ and $\phi$, one obtains

$$
\begin{aligned}
B^{2} \frac{\partial}{\partial \chi} \frac{e n v_{\|}}{c B}= & \left(\frac{\partial}{\partial \vartheta} \frac{1}{B^{2}}\right) \frac{\partial p}{\partial \psi} \nabla \vartheta \cdot \nabla \psi \times \nabla \chi+\left(\frac{\partial}{\partial \psi} \frac{1}{B^{2}}\right) \frac{\partial p}{\partial \vartheta} \nabla \psi \cdot \nabla \vartheta \times \nabla \chi+\frac{1}{B^{2}} \frac{\partial^{2} p}{\partial \vartheta \partial \psi} \nabla \vartheta \cdot \nabla \psi \times \nabla \chi \\
& +\frac{1}{B^{2}} \frac{\partial^{2} p}{\partial \psi \partial \vartheta} \nabla \psi \cdot \nabla \vartheta \times \nabla \chi+\frac{1}{B^{2}} \frac{\partial^{2} p}{\partial \phi \partial \psi} \nabla \phi \cdot \nabla \psi \times \nabla \chi+\frac{1}{B^{2}} \frac{\partial^{2} p}{\partial \phi \partial \vartheta} \nabla \phi \cdot \nabla \vartheta \times \nabla \chi
\end{aligned}
$$

Since $\nabla \psi \cdot \nabla \vartheta \times \nabla \chi=B^{2}$ and $\nabla \vartheta \cdot \nabla \psi \times \nabla \chi=-B^{2}$, the third and fourth terms in the last equation cancel each other. In the fifth term one can change the order of the partial derivatives over $\phi$ and $\psi$ and then make use of the equality $\partial p / \partial \phi$ $=-e p / T$. The triple product $\nabla \phi \cdot \nabla \psi \times \nabla \chi$ is equal to $(\partial \phi / \partial \vartheta) \nabla \vartheta \cdot \nabla \psi \times \nabla \chi=-B^{2}(\partial \phi / \partial \vartheta)$. The sixth term is transformed in a similar way. Dividing both sides of the last equation by $B^{2}$ leads to

$$
\begin{aligned}
\frac{\partial}{\partial \zeta} \frac{e n v_{\|}}{c B}= & -\frac{\partial p}{\rho \partial \rho} \frac{\partial}{\partial \vartheta} \frac{1}{B^{2}}+\frac{\partial p}{\partial \vartheta} \frac{\partial}{\rho \partial \rho} \frac{1}{B^{2}}+\frac{1}{B^{2}} \frac{\partial e \phi}{\partial \vartheta} \frac{\partial}{\rho \partial \rho} \frac{p}{T} \\
& -\frac{1}{B^{2}} \frac{\partial e \phi}{\rho \partial \rho} \frac{\partial}{\partial \vartheta} \frac{p}{T},
\end{aligned}
$$

where the use of $\rho$ and $\zeta$ (instead of $\psi$ and $\chi$ ) has been restored. This equation allows us to calculate the plasma current along the magnetic field lines. Since the current 
vanishes at the ends of the plasma column, the integral of the right-hand side over the entire range of $\zeta$ must be equal to zero. This yields the "solvability condition"

$$
\begin{aligned}
0= & \int_{-\infty}^{\infty}\left\{-\frac{\partial p}{\partial \rho} \frac{\partial}{\partial \vartheta} \frac{1}{B^{2}}+\frac{\partial p}{\partial \vartheta} \frac{\partial}{\partial \rho} \frac{1}{B^{2}}+\frac{1}{B^{2}} \frac{\partial e \phi}{\partial \vartheta} \frac{\partial}{\partial \rho} \frac{p}{T}\right. \\
& \left.-\frac{1}{B^{2}} \frac{\partial e \phi}{\partial \rho} \frac{\partial}{\partial \vartheta} \frac{p}{T}\right\} d \zeta,
\end{aligned}
$$

where the integration is formally extended over an infinite interval (actually it covers the interval of a magnetic field line where the plasma pressure $p$ is nonzero).

The constraint (40) interrelates two functions of $\rho$ and $\vartheta$, namely $N$ and $T$, and, in general, it allows determining $N(\rho, \vartheta)$ if $T(\rho, \vartheta)$ is given or vice versa. One can argue, however, that $T(\rho, \vartheta)$ is not completely independent of $N(\rho, \vartheta)$. Indeed, a differential plasma rotation would result in a fast sharpening of the temperature gradient across the plasma streamlines so that even a weak transverse thermal conductivity effectively flattens the temperature along the streamlines. Therefore one can assume that $\mathbf{v} \cdot \nabla T=0$ in addition to the condition $\mathbf{B} \cdot \nabla T=0$ used in the derivation of Eq. (30). Dotting Eq. (35) with $\nabla T$ one obtains the relation

$$
\frac{\partial N}{\partial \rho} \frac{\partial T}{\partial \vartheta}-\frac{\partial N}{\partial \vartheta} \frac{\partial T}{\partial \rho}=0 .
$$

This means that $T$ depends on $\rho$ and $\vartheta$ through the dependence of $N$ on these coordinates, i.e.,

$$
T(\rho, \vartheta)=T[N(\rho, \vartheta)] .
$$

In the following, the stronger assumption of a constant temperature $T$ is adopted, which is relevant to the state of global thermal equilibrium. ${ }^{26,27}$ This state is also characterized by a rigid plasma rotation and will be analyzed in details in Secs. VIII B and IX B. For $T=$ const, Eq. (40) reduces to the simpler form

$$
\begin{aligned}
& \frac{\partial N}{\partial \vartheta} \int_{-\infty}^{\infty}\left\{\frac{\partial}{\partial \rho} \frac{1}{B^{2}}-\frac{1}{B^{2}} \frac{\partial}{\partial \rho} \frac{e \phi}{T}\right\} \mathrm{e}^{-e \phi / T} d \zeta \\
& =\frac{\partial N}{\partial \rho} \int_{-\infty}^{\infty}\left\{\frac{\partial}{\partial \vartheta} \frac{1}{B^{2}}-\frac{1}{B^{2}} \frac{\partial}{\partial \vartheta} \frac{e \phi}{T}\right\} e^{-e \phi / T} d \zeta,
\end{aligned}
$$

where it has been taken into account that $N(\rho, \vartheta)$ does not depend on $\zeta$. Note again that the exponential $e^{-e \phi / T}$ vanishes at the ends of the plasma column, so the integration is extended over an infinite interval only formally.

Considering now the case of a weak magnetic perturbation, one can assume that $N(\rho, \vartheta)=N_{0}(\rho)+\epsilon N_{1}(\rho, \vartheta)$ in analogy with the ordering $\phi(\rho, \vartheta, \zeta)=\phi_{0}(\rho, \zeta)+\epsilon \phi_{1}(\rho, \vartheta, \zeta)$. Linearizing Eq. (43) over $\epsilon$ gives the relation

$$
\begin{aligned}
& \frac{\partial N_{1}}{\partial \vartheta} \int_{-\infty}^{\infty}\left\{\frac{\partial}{\partial \rho} \frac{1}{B_{0}^{2}}-\frac{1}{B_{0}^{2}} \frac{\partial}{\partial \rho} \frac{e \phi_{0}}{T}\right\} e^{-e \phi_{0} / T} d \zeta \\
& =\frac{\partial N_{0}}{\partial \rho} \int_{-\infty}^{\infty}\left\{\left(\frac{\partial}{\partial \vartheta} \frac{1}{B^{2}}\right)_{1}-\frac{1}{B_{0}^{2}} \frac{\partial}{\partial \vartheta} \frac{e \phi_{1}}{T}\right\} \mathrm{e}^{-e \phi_{0} / T} d \zeta .
\end{aligned}
$$

It is shown below that the first terms in the integrands on both sides of the last equation can be neglected in most situations, so that this constraint can be further simplified.

Finally, it is worth noting that the separation of $N(\rho, \vartheta)$ in an "axisymmetric," $N_{0}(\rho)$, and a "perturbed" part, $N_{1}(\rho, \vartheta)$, is not unique. Generally, a supplementary condition has to be imposed in order to eliminate this uncertainty. In particular, it is shown below that in many circumstances the "perturbed" part $N_{1}(\rho, \vartheta)$ can be set to zero (with a suitable choice of the origin of the flux system of coordinates), a condition which greatly simplifies the search of plasma equilibrium under the effect of weak inhomogeneity of the magnetic field.

\section{POISSON'S EQUATION IN CURVILINEAR COORDINATES}

In a general system of curvilinear coordinates Poisson's equation takes the form

$$
\frac{1}{\sqrt{g}} \frac{\partial}{\partial \xi^{i}} \sqrt{g} g^{i k} \frac{\partial \phi}{\partial \xi^{k}}=-4 \pi e n .
$$

With the adopted flux coordinates $\xi^{i}=(\rho, \vartheta, \zeta)$ the only nonzero elements of $\sqrt{g} g^{i k}$ are

$\sqrt{g} g^{11}=g_{22} g_{33} / \sqrt{g}=g_{22} / \rho, \quad \sqrt{g} g^{22}=g_{11} g_{33} / \sqrt{g}=g_{11} / \rho$,

$\sqrt{g} g^{12}=-g_{21} g_{33} / \sqrt{g}=-g_{21} / \rho, \quad \sqrt{g} g^{33}=\left(g_{11} g_{22}-g_{12}^{2}\right) / \sqrt{g}=\rho$.

The Poisson equation therefore reduces to

$$
\begin{aligned}
\frac{1}{\sqrt{g}} \frac{\partial}{\partial \rho} \frac{g_{22}}{\rho} \frac{\partial \phi}{\partial \rho}+\frac{1}{\sqrt{g}} \frac{\partial}{\partial \vartheta} \frac{g_{11}}{\rho} \frac{\partial \phi}{\partial \vartheta}+\frac{1}{\sqrt{g}} \frac{\partial^{2} \phi}{\partial \zeta^{2}}-\frac{1}{\sqrt{g}} \frac{\partial}{\partial \rho} \frac{g_{21}}{\rho} \frac{\partial \phi}{\partial \vartheta} \\
-\frac{1}{\sqrt{g}} \frac{\partial}{\partial \vartheta} \frac{g_{12}}{\rho} \frac{\partial \phi}{\partial \rho}=-4 \pi e n .
\end{aligned}
$$

For a weakly nonuniform magnetic field, the off-diagonal element, $g_{12}=g_{21}$, of the metric tensor turns out to be small, being linear over the amplitude $\epsilon$ of the inhomogeneity. If one seeks for a solution of Eq. (46) in the form $\phi$ $=\phi_{0}(\rho, \zeta)+\epsilon \phi_{1}(\rho, \vartheta, \zeta)$, where the second term represents a small correction linear in $\epsilon$, then it is found that the fourth term in the left-hand side of Eq. (46) can be dropped, and only $\phi_{0}$ should be kept in the last term. Using the metric tensor (27), Eq. (46) can be cast into the form

$$
\begin{gathered}
\nabla^{\prime 2}\left(\phi_{0}+\epsilon \phi_{1}\right)-2 \epsilon \frac{\partial \rho_{1}}{\partial \rho} \frac{\partial^{2} \phi_{0}}{\partial \rho^{2}}-2 \epsilon\left(\nabla^{\prime 2} \rho_{1}+\frac{1}{\rho^{2}} \rho_{1}\right) \frac{1}{\rho} \frac{\partial \phi_{0}}{\partial \rho} \\
-2 \epsilon \frac{\partial \zeta_{1}}{\partial \zeta} \frac{\partial^{2} \phi_{0}}{\partial \zeta^{2}}=-4 \pi e\left(n_{0}+\epsilon n_{1}\right),
\end{gathered}
$$

where

$$
\nabla^{\prime 2} \equiv \frac{1}{\rho} \frac{\partial}{\partial \rho} \rho \frac{\partial}{\partial \rho}+\frac{1}{\rho^{2}} \frac{\partial^{2}}{\partial \vartheta^{2}}+\frac{\partial^{2}}{\partial \zeta^{2}}
$$

is the standard Laplace operator in cylindrical coordinates. Equation (47) was first derived by O'Neil. ${ }^{18}$ It can be written into several equivalent forms using the equality 


$$
\nabla^{\prime 2} \rho_{1}+\frac{1}{\rho^{2}} \rho_{1}=\frac{2}{\rho}\left(\frac{\partial \zeta_{1}}{\partial \zeta}-\frac{\partial \rho_{1}}{\partial \rho}+F\right),
$$

which is demonstrated in Appendix A. Here $F=F(\rho, \vartheta)$ is a function related to $\rho_{10}$. In what follows it is always assumed to be equal to zero.

\section{WEAK MAGNETIC SQUEEZE}

As a first example of the use of the formalism developed in Secs. III-V the case of a magnetic squeeze $(m=0)$ is considered. It is shown in particular that in the lowest order of the paraxial approximation the main results of Ref. 14 are recovered.

It is worth nothing that in general the computation of the metric tensor in the paraxial approximation requires some care. If the determinant $g$ is to be evaluated up to terms of order $\rho^{n}$, all terms up to order $\rho^{n}$ have to be retained in $\zeta_{1}$ when computing $\rho_{1}$ and $\vartheta_{1}$, otherwise all the elements of the tensor except $g_{33}$ will be calculated with insufficient accuracy.

Inserting

$\rho_{1}=-\frac{\delta B_{0}}{2 B_{*}} \rho, \quad \vartheta_{1}=0, \quad \zeta_{1}=-\int_{\zeta_{0}}^{\zeta} \frac{\delta B_{0}}{B_{*}} d \zeta+\frac{\delta B_{0}^{\prime}}{4 B_{*}} \rho^{2}$

(with $\delta B_{0}=B_{0}-B_{*}$ ) into Eq. (27) yields

$$
\begin{aligned}
& g_{11}=1-\epsilon \delta B_{0} / B_{*}, \quad g_{12}=0, \\
& g_{22}=\left(1-\epsilon \delta B_{0} / B_{*}\right) \rho^{2}, \quad g_{33}=1-\epsilon 2 \delta B_{0} / B_{*},
\end{aligned}
$$

where $g_{33}$ has been consistently truncated to the order $\rho^{0}$.

For an axisymmetric perturbation Eq. (44) reduces to $\partial N_{1} / \partial \vartheta=0$. Then

$$
n_{0}(\rho, \zeta)=N_{0}(\rho) \exp \left[-e \phi_{0}(\rho, \zeta) / T\right]
$$

and

$$
n_{1}(\rho, \zeta)=-n_{0}(\rho, \zeta) \frac{e \phi_{1}(\rho, \zeta)}{T}
$$

as it has been adopted in Ref. 14 .

Using Eqs. (49) in Eq. (47) gives

$$
\begin{aligned}
& \nabla^{\prime 2}\left(\phi_{0}+\epsilon \phi_{1}\right)+\epsilon \frac{\delta B_{0}}{B_{*}} \frac{1}{\rho} \frac{\partial}{\partial \rho} \rho \frac{\partial \phi_{0}}{\partial \rho}+2 \epsilon \frac{\delta B_{0}}{B_{*}} \frac{\partial^{2} \phi_{0}}{\partial \zeta^{2}} \\
& =-4 \pi e\left(n_{0}+\epsilon n_{1}\right) .
\end{aligned}
$$

For the case of an infinitely long plasma column considered in Ref. 14, where $\phi_{0}=\phi_{0}(\rho)$ and $n_{0}=n_{0}(\rho)$ do not depend on $\zeta$, the last term in the left-hand side of Eq. (53) can be dropped leading to the following equations:

$$
\begin{aligned}
& \frac{1}{\rho} \frac{d}{d \rho} \rho \frac{d \phi_{0}}{d \rho}=-4 \pi e n_{0}, \\
& \frac{1}{\rho} \frac{\partial}{\partial \rho} \rho \frac{\partial \phi_{1}}{\partial \rho}+\frac{\partial^{2} \phi_{1}}{\partial \zeta^{2}}=4 \pi e n_{0}\left[\frac{\delta B_{0}}{B_{*}}+\frac{e \phi_{1}(\rho, \zeta)}{T}\right]
\end{aligned}
$$

for the unperturbed and perturbed parts of the electric potential, respectively.
Equation (55) has been analyzed in Ref. 14 in a longthin approximation neglecting all derivatives over $\zeta$. Corrections of order $\epsilon \times(a / L)^{2}$ could be included by keeping the terms of order $\delta B_{0}^{\prime \prime} \rho^{3}$ in $\rho_{1}$ and $\delta B_{0}^{\prime \prime \prime} \rho^{4}$ in $\zeta_{1}$ in Eq. (49). However this does not lead to any qualitatively new effect with respect to the results reported in Ref. 14.

\section{MAGNETIC TILT: PRELIMINARY TREATMENT}

In this section a preliminary analysis of the magnetic tilt, $m=1$, is performed and some formulas are obtained, which are then used in the next two sections where analytically solvable examples of plasma equilibrium in a tilted magnetic field are presented.

$$
\begin{aligned}
& \rho_{1}=\left[\int_{\zeta_{0}}^{\zeta} \frac{B_{1 x}}{B_{*}} d \zeta-\frac{3 B_{1 x}^{\prime}}{8 B_{*}} \rho^{2}\right] \cos \vartheta, \\
& \vartheta_{1}=\left[-\frac{1}{\rho} \int_{\zeta_{0}}^{\zeta} \frac{B_{1 x}}{B_{*}} d \zeta+\frac{B_{1 x}^{\prime}}{8 B_{*}} \rho^{2}\right] \sin \vartheta, \\
& \zeta_{1}=\left[-\frac{B_{1 x}}{B_{*}}+\frac{B_{1 x}^{\prime \prime}}{8 B_{*}} \rho^{2}\right] \rho, \cos \vartheta,
\end{aligned}
$$

into Eq. (27) yields

$$
\begin{aligned}
& g_{11}=1-\epsilon \frac{3 B_{1 x}^{\prime}}{2 B_{*}} \rho \cos \vartheta, \quad g_{12}=\epsilon \frac{B_{1 x}^{\prime}}{2 B_{*}} \rho^{2} \sin \vartheta \\
& g_{22}=\left(1-\epsilon \frac{B_{1 x}^{\prime}}{2 B_{*}} \rho \cos \vartheta\right) \rho^{2}, \quad g_{33}=1-\epsilon \frac{2 B_{1 x}^{\prime}}{B_{*}} \rho \cos \vartheta
\end{aligned}
$$

where the element $g_{33}$ has been truncated to the order $\rho^{1}$. Gathering $\rho_{1}$ and $\zeta_{1}$ into Eq. (47), the Poisson equation for the case of a magnetic tilt is obtained,

$$
\begin{aligned}
& \nabla^{\prime 2}\left(\phi_{0}+\epsilon \phi_{1}\right)+\epsilon \frac{B_{1 x}^{\prime}}{B_{*}}\left[\frac{3}{2} \frac{\partial^{2} \phi_{0}}{\partial \rho^{2}}+\frac{1}{2 \rho} \frac{\partial \phi_{0}}{\partial \rho}+2 \frac{\partial^{2} \phi_{0}}{\partial \zeta^{2}}\right] \rho \cos \vartheta \\
& =-4 \pi e\left(n_{0}+\epsilon n_{1}\right) .
\end{aligned}
$$

Equation (58) must be supplemented with the boundary condition at the wall of the conducting chamber surrounding the plasma column. In flux coordinates the inner surface of the wall is described by the equation $\rho=R-\epsilon \rho_{1}(\rho, \vartheta, \zeta)$, where $R$ denotes the inner radius of the wall. Expanding the exact boundary condition $\left(\phi_{0}+\epsilon \phi_{1}\right)=0$ at this value of $\rho$ around $\rho=R$ yields the boundary condition in the form

$$
\begin{aligned}
& \phi_{0}(R, \vartheta)=0 \\
& \phi_{1}(R, \vartheta, \zeta)=\frac{\partial \phi_{0}(R, \zeta)}{\partial R} \rho_{1}(R, \vartheta, \zeta)
\end{aligned}
$$

The parallel current constraint (44) can also be simplified when taking into account the specific features of the tilt perturbations. Noting that $1 /|\mathbf{B}|^{2}=g_{33} / B_{*}^{2}$, as it follows from Eqs. (9) and (10), and using $g_{33}$ from Eq. (57), one finds that 


$$
\frac{1}{B^{2}}=\frac{1}{B_{*}^{2}}\left\{1+2 \epsilon\left[-\frac{B_{1 x}^{\prime}}{B_{*}} \rho \cos \vartheta\right]\right\} .
$$

Hence,

$$
\frac{\partial}{\partial \vartheta} \frac{1}{B^{2}}=\frac{2 \epsilon}{B_{*}^{2}} \frac{B_{1 x}^{\prime}}{B_{*}} \rho \sin \vartheta
$$

and

$$
\frac{\partial}{\partial \rho} \frac{1}{B^{2}}=\frac{2 \epsilon}{B_{*}^{2}}\left[-\frac{B_{1 x}^{\prime}}{B_{*}} \cos \vartheta\right]
$$

This means that for a uniform magnetic tilt, such that $B_{1 x}^{\prime}(\zeta)=0$ everywhere, Eq. (44) reduces to

$N_{1}=\frac{\partial N_{0}}{\partial \rho} \int_{-\infty}^{\infty} \frac{e \phi_{1}}{T} e^{-e \phi_{0} / T} d \zeta / \int_{-\infty}^{\infty}\left(\frac{\partial}{\partial \rho} \frac{e \phi_{0}}{T}\right) \mathrm{e}^{-e \phi_{0} / T} d \zeta$.

This expression remains approximately valid if the first terms on both sides of Eq. (44) are small in comparison with the second terms. This condition is satisfied in a long-thin approximation, because dropping these terms is equivalent to dropping all derivatives over $\zeta$, as it was shown above.

A careful treatment reveals that the first terms on both sides of Eq. (44) are indeed small in the sense indicated above if

$$
\left(a / \lambda_{D}\right)(L / R)^{2} \gg 1
$$

This condition holds for virtually all ongoing non-neutral plasma experiments, since usually $a \geqslant \lambda_{D}$ and $L \gg R$.

To derive the condition (64), one may observe that the first term in curly braces on the right-hand side of Eq. (44) can be estimated as

$$
\frac{\partial}{\partial \vartheta} \frac{1}{B^{2}} \sim \frac{1}{B^{2}} \frac{\epsilon a}{L},
$$

if the ordering $\epsilon B_{1 x}^{\prime} / B_{*} \sim \epsilon / L$ is used in Eq. (61) and $\epsilon B_{1 x} / B_{*} \sim \epsilon$ denotes the angle of the magnetic tilt. The second term on the right-hand side

$$
\frac{1}{B^{2}} \frac{\partial}{\partial \vartheta} \frac{e \phi_{1}}{T} \sim \frac{1}{B^{2}} \frac{e \phi_{1}}{T},
$$

can be evaluated by first taking into account that a dipole perturbation of the magnetic field causes a displacement of the plasma column from the geometrical axis of the device of the order of $\epsilon \zeta \sim \epsilon L$. The displaced plasma column induces an image charge on the cylindrical conducting wall of the device, and the image charge produces a quasiuniform electric field $E_{1} \sim\left(\pi a^{2} e n / R^{2}\right) \epsilon L$ at the location of the plasma column. The perturbation of the electric potential is then evaluated as $\phi_{1} \approx E_{1} \times \lambda_{D}$, since the field is shielded within a narrow edge layer of the plasma column with a thickness of the order of few $\lambda_{D}$. Hence,

$$
\frac{e \phi_{1}}{T} \sim \frac{a^{2}}{R^{2}} \frac{\epsilon L}{\lambda_{D}}
$$

$$
\frac{1}{B^{2}} \frac{\partial}{\partial \vartheta} \frac{e \phi_{1}}{T} \sim \frac{1}{B^{2}} \frac{a^{2}}{R^{2}} \frac{\epsilon L}{\lambda_{D}} .
$$

The comparison of Eqs. (65) and (67) leads to the inequality (64) as the condition for the expression (63) to be valid.

Comparing similar terms on the left-hand side of Eq. (44) gives a less stringent condition since the first term

$$
\frac{\partial}{\partial \rho} \frac{1}{B^{2}} \sim \frac{1}{B^{2}} \frac{\epsilon}{L}
$$

is proportional to $\epsilon$, whereas the second one,

$$
\frac{1}{B^{2}} \frac{\partial}{\partial \rho} \frac{e \phi_{0}}{T} \sim \frac{1}{B^{2}} \frac{a}{\lambda_{D}^{2}},
$$

is not.

A brief survey of the equations presented in this section shows that they acquire an extremely simple form if the magnetic tilt is uniform, i.e., if $B_{1 x}^{\prime}(\zeta)=0$ everywhere. In particular, the magnetic field does not explicitly appear in the metric tensor (57) and in the Poisson equation (58). Indeed, considering cylindrical coordinates

$$
\begin{aligned}
& \rho=\sqrt{(r \cos \theta \cos \epsilon-z \sin \epsilon)^{2}+(r \sin \theta)^{2}}, \\
& \vartheta=\arctan [r \cos \theta \cos \epsilon-z \sin \epsilon, r \sin \theta], \\
& \zeta=r \cos \theta \sin \epsilon+z \cos \epsilon,
\end{aligned}
$$

with the $\zeta$-axis directed along the magnetic field, which is uniformly tilted by the angle $\epsilon$ with respect to the other cylindrical system of coordinates $(r, \theta, z)$ with the $z$-axis along the symmetry axis of the device, one can readily calculate the exact form of the metric tensor,

$$
g_{i k}=\left[\begin{array}{ccc}
1 & 0 & 0 \\
0 & \rho^{2} & 0 \\
0 & 0 & 1
\end{array}\right] .
$$

Due to the extreme simplicity of the resulting equations, the special case of uniform magnetic tilt is treated in details in the next section.

\section{UNIFORM MAGNETIC TILT}

In this section the equilibrium of a long plasma column immersed in a uniform magnetic field slightly tilted with respect to the axis of the cylindrical conducting chamber is analyzed. Let $\alpha \ll 1$ denote the tilt angle,

$$
\frac{B_{1 x}(\zeta)}{B_{*}} \equiv \alpha
$$

The constancy of $\alpha$ greatly simplifies the search for the plasma equilibrium.

First of all, the expression (63) becomes equivalent to Eq. (44). Second, the perturbed quantities,

$$
\zeta_{1}=-\alpha \rho \cos \vartheta
$$

and 


$$
\rho_{1}=\alpha\left(\zeta-\zeta_{0}\right) \cos \vartheta
$$

acquire an extremely simple form. The same is true for the Poisson equation for the perturbed part of the potential [cf. it with Eq. (58)],

$$
\frac{1}{\rho} \frac{\partial}{\partial \rho} \rho \frac{\partial \phi_{1}}{\partial \rho}+\frac{1}{\rho^{2}} \frac{\partial^{2} \phi_{1}}{\partial \vartheta^{2}}+\frac{\partial^{2} \phi_{1}}{\partial \zeta^{2}}=4 \pi e\left[N_{0} \frac{e \phi_{1}}{T}-N_{1}\right] e^{-e \phi_{0} / T} \text {. }
$$

Since Eq. (71) does not contain the tilt angle $\alpha$ explicitly, the perturbed geometry of the magnetic field enters the problem only through the boundary condition (59b), which simply reads

$$
\phi_{1}(R, \vartheta, \zeta)=\alpha\left(\zeta-\zeta_{0}\right) \frac{\operatorname{Ad} \phi_{0}(R)}{d R} \cos \vartheta
$$

The specific form Eq. (73) of the boundary condition suggests that $\phi_{1}$ could be a linear function of $\zeta$, at least in the central part of the plasma column, sufficiently far from the column ends, where the unperturbed part of the electric potential is constant along the magnetic field lines, i.e., $\phi_{0}$ $=\phi_{0}(\rho)$.

When a perturbed potential of the form

$$
\phi_{1}(\rho, \zeta, \vartheta)=\phi_{1}^{(1)}(\rho) \frac{\alpha\left(\zeta-\zeta_{0}\right)}{R} \cos \vartheta
$$

is considered, the last term in the left-hand side of Eq. (71) vanishes but one has to be reminded that $\phi_{1}$ also determines the perturbed function $N_{1}$ in the right-hand side of Eq. (71). It is evident, however, that the right-hand side of Eq. (63) can be made equal to zero by a proper choice of the free parameter $\zeta_{0}$, not discussed so far, thus resulting in $N_{1}=0$.

Indeed, taking into account the specific form Eq. (70) of $\rho_{1}$ for the uniform magnetic tilt, $\zeta_{0}$ turns out to be the position of crossing between the device axis $z(r=0)$ and the plasma column axis $\zeta(\rho=0)$. In other words, $N_{1}$ can be set to zero by a proper choice of the origin of the curvilinear system of coordinates $(\rho, \vartheta, \zeta)$. This option is used in the following treatment. For the same reason it is possible to set $\zeta_{0}=0$ without loss of generality.

With $N_{1}=0$ and $\partial^{2} \phi_{1} / \partial \zeta^{2}=0$, the Poisson equation (71) for the radial part $\phi_{1}^{(1)}(\rho)$ of the perturbed potential $\phi_{1}(\rho, \vartheta, \zeta)$ reduces to

$$
\frac{1}{\rho} \frac{d}{d \rho} \rho \frac{d \phi_{1}^{(1)}}{d \rho}-\frac{1}{\rho^{2}} \phi_{1}^{(1)}=\frac{4 \pi e^{2} n_{0}}{T} \phi_{1}^{(1)},
$$

where $n_{0}=n_{0}(\rho)=N_{0}(\rho) e^{-e \phi_{0} / T}$, and the boundary condition (72) reads

$$
\phi_{1}^{(1)}(R)=R \frac{d \phi_{0}(R)}{d R} .
$$

As for the unperturbed potential $\phi_{0}$, it obeys the equation

$$
\frac{1}{\rho} \frac{d}{d \rho} \rho \frac{d}{d \rho} \phi_{0}=-4 \pi e n_{0}
$$

with the boundary condition (59a), under the same assumptions, i.e., sufficiently far from the plasma column ends.

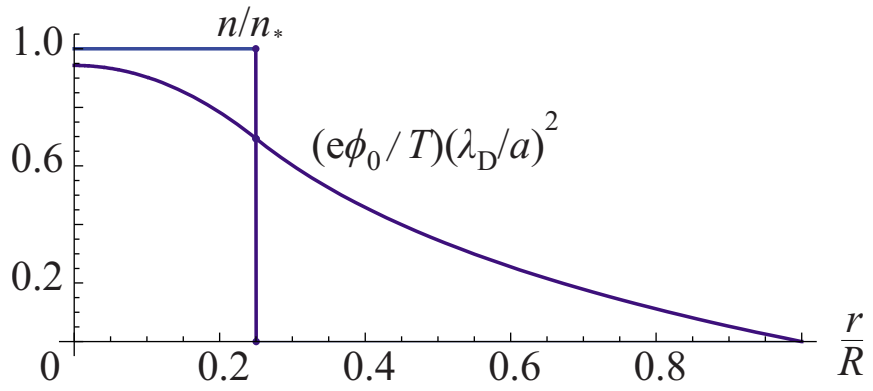

FIG. 2. (Color online) Model of a non-neutral plasma column with a stepwise density profile: Radial profiles of plasma density $n$ and electrostatic potential $\phi_{0}$. The ratio $a / R=0.25$ between the plasma radius and the wall radius is typical for the Eltrap experiment (Ref. 28).

\section{A. Stepwise density profile}

A simple analytical solution of the systems (74)-(76) exists for a stepwise density profile

$$
n_{0}(\rho)=n_{*} H(a-\rho),
$$

where $H$ is the Heaviside step function, and $a$ is the unperturbed radius of the plasma column. In this case, the solution of Eq. (75) takes the form

$$
\frac{e \phi_{0}(\rho)}{T}=\frac{a^{2}-\rho^{2}}{4 \lambda_{D}^{2}}+\frac{a^{2}}{2 \lambda_{D}^{2}} \ln \frac{R}{a}
$$

inside the plasma column $(\rho \leqslant a)$, and

$$
\frac{e \phi_{0}(\rho)}{T}=\frac{a^{2}}{2 \lambda_{D}^{2}} \ln \frac{R}{\rho}
$$

outside (for $\rho>a$ ). Note that the Debye radius $\lambda_{D}$ $=\sqrt{T / 4 \pi e^{2} n_{*}}$ is determined here by the density $n_{*}$ at the column axis; the same rule will also be used in the subsequent sections when smooth plasma density profiles will be considered. Typical radial profiles of density, electric potential, angular rotation frequency $\omega$ and radial electric field are drawn in Fig. 2.

Solving Eq. (74) yields the perturbed potential

$$
\frac{e \phi_{1}^{(1)}}{T}=-\frac{2 a \lambda_{D} I_{1}\left(\rho / \lambda_{D}\right)}{\left(R^{2}-a^{2}\right) I_{0}\left(a / \lambda_{D}\right)+2 a \lambda_{D} I_{1}\left(a / \lambda_{D}\right)} \frac{a R}{2 \lambda_{D}^{2}}
$$

for $\rho<a$, and

$$
\frac{e \phi_{1}^{(1)}}{T}=-\frac{\left(\rho^{2}-a^{2}\right) I_{0}\left(a / \lambda_{D}\right)+2 a \lambda_{D} I_{1}\left(a / \lambda_{D}\right)}{\left(R^{2}-a^{2}\right) I_{0}\left(a / \lambda_{D}\right)+2 a \lambda_{D} I_{1}\left(a / \lambda_{D}\right)} \frac{a^{2} R}{2 \rho \lambda_{D}^{2}}
$$

for $\rho>a$, where $I_{n}$ are the modified Bessel functions of the first kind of order $n$. These solutions (79a) and (79b) are drawn in Fig. 3(a).

In the cold plasma case, $a \gg \lambda_{D}$, the previous formulas simplify to

$$
\frac{e \phi_{1}^{(1)}}{T}=-\frac{a^{2}}{R^{2}-a^{2}} \sqrt{\frac{a}{\rho}} \exp \left(\frac{\rho-a}{\lambda_{D}}\right) \frac{R}{\lambda_{D}}
$$

close to the plasma edge, $\lambda_{D} \ll \rho \leqslant a$, and 

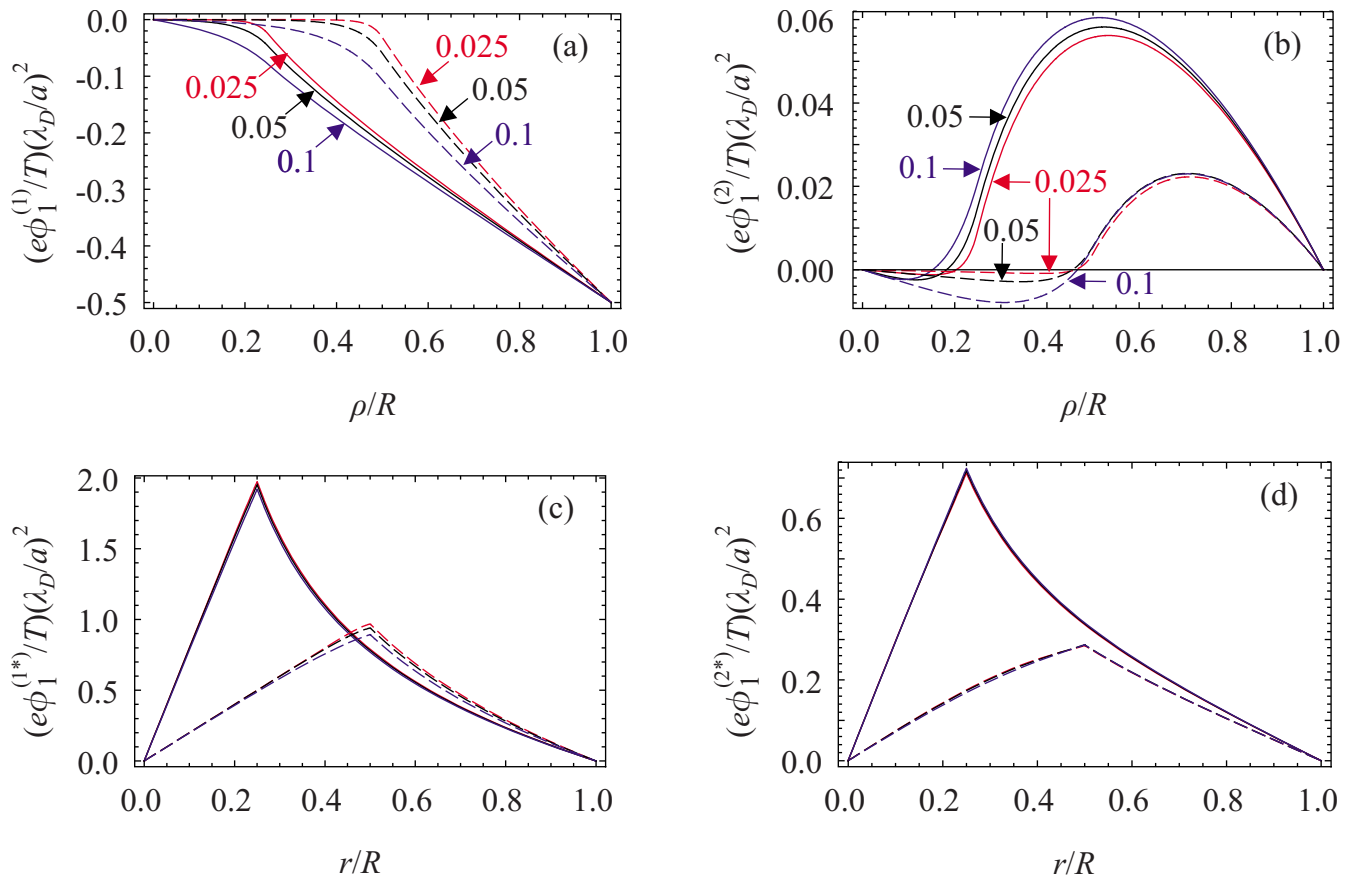

FIG. 3. (Color online) Perturbed potentials $\phi_{1}^{(1)}(\rho), \phi_{1}^{(2)}(\rho)$ in flux coordinates (graphs a and b, respectively) and the same profiles $\phi_{1}^{(1 *)}(r)$, $\phi_{1}^{(2 *)}(r)$ in device coordinates (graphs $\mathrm{c}$ and $\mathrm{d}$ ). The plots are drawn for two values of the plasma radius, $a / R=0.25$ (solid curves) and $a / R=0.5$ (dashed curves), and for three values of the Debye length, $\lambda_{D} / R=0.025$ (red), 0.05 (black), and 0.1 (blue).

$$
\frac{e \phi_{1}^{(1)}}{T}=-\frac{\left(R^{2}-\rho^{2}\right) a^{3} R}{\left(R^{2}-a^{2}\right)^{2} \rho \lambda_{D}}-\frac{\left(\rho^{2}-a^{2}\right) a^{2} R}{2\left(R^{2}-a^{2}\right) \rho \lambda_{D}^{2}}
$$

for $\rho>a$. These expressions show that the perturbed potential inside the plasma column is typically $a / \lambda_{D}$ times smaller than outside of it [note that Eq. (80b) contains a term proportional to $1 / \lambda_{D}^{2}$ which vanishes at the plasma boundary, whereas Eq. (80a) has a term proportional to $\left.1 / \lambda_{D}^{1}\right]$. Within the plasma column the perturbation is localized in a narrow layer of the order of a few Debye lengths from the column edge. The estimate (66) obtained in Sec. VII from elementary arguments refers to this edge layer. More precisely, the perturbation exponentially vanishes towards the column axis as

$$
\frac{e \phi_{1}^{(1)}}{T}=-\sqrt{\frac{\pi}{2}}\left(\frac{a}{\lambda_{D}}\right)^{5 / 2} \frac{\rho R}{R^{2}-a^{2}} \exp \left(-\frac{a}{\lambda_{D}}\right)
$$

The boundary perturbation is therefore effectively shielded at the edge of the plasma column, a fact that was already emphasized in Ref. 14 for a "potential squeeze." In Sec. IX it will be shown that in the case of a variable magnetic tilt the electric potential is instead non-negligibly perturbed in the plasma interior, showing a relation between the magnetic field strength and the electric potential perturbation very similar to that found for a magnetic squeeze. ${ }^{14}$

The function $\phi_{1}(\rho, \vartheta, \zeta)$ characterizes the variation of the electric potential along a magnetic field line fixed by given flux coordinates $\rho$ and $\vartheta$; it therefore describes the trapping of particles with small longitudinal velocity. When moving along a magnetic field line, a particle undergoes a smoothly varying electric potential $\phi_{1}(\rho, \vartheta, \zeta)$, although the magnetic field strength $B$ remains unchanged. The constancy of $B$ distinguishes the present case from that of a magnetic squeeze analyzed in Ref. 14, so that only potentially trapped particles exist in the case of a uniformly tilted magnetic field perturbation whereas a magnetic squeeze gives rise also to a population of magnetically trapped particles.

When perturbed quantities are computed in the natural cylindrical coordinates related to the confinement device, one needs to take into account the displacement of the magnetic field lines. In particular, the electric potential is given by the function

$$
\phi(r, \theta, z) \approx \phi_{0}\left(r-\epsilon \rho_{1}\right)+\epsilon \phi_{1}(r, \theta, z) .
$$

Retaining all terms linear in $\epsilon$, one finds

$$
\phi_{1}^{*}(r, \theta, z)=-\phi_{0}^{\prime}(r) \rho_{1}(r, \theta, z)+\phi_{1}(r, \theta, z),
$$

representing the variation of the electric potential along a straight line parallel to the device axis $z$. The first term in the right-hand side of Eq. (81) cancels the second one at the conducting wall, so that $\phi_{1}^{*}(R, \theta, z)=0$, but it is approximately $a / \lambda_{D}$ times larger than the second term at the column edge, where $\phi_{1}^{*} \gg \phi_{1}$. However, the correction of the electric potential related to the first term in Eq. (81) does not affect the particle motion along a magnetic field line. A similar picture was found for the case of a magnetic squeeze. ${ }^{14}$ The radial profile 

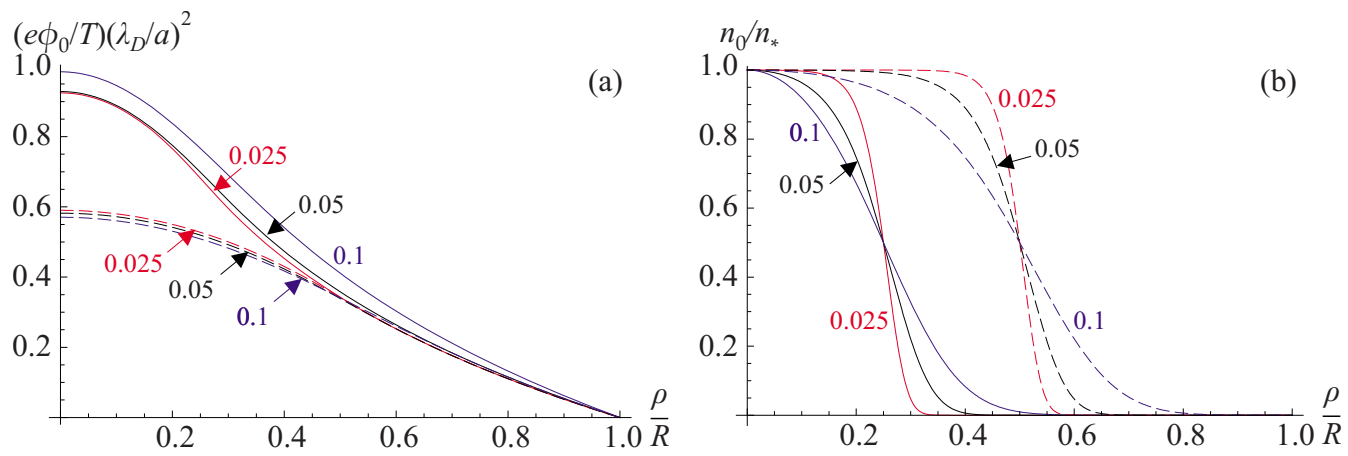

FIG. 4. (Color online) Thermal equilibrium potential $\phi_{0}$ (a) and plasma density $n_{0}$ (b) for a fixed plasma radius (evaluated at the level $n_{0} / n_{*}=1 / 2$ ) $a$ $=1.125 \mathrm{~cm}$ (solid curves) and $a=2.25 \mathrm{~cm}$ (dashed curves), and three different ratios $\lambda_{D} / R=0.025$ (red), 0.05 (black), and 0.1 (blue), corresponding to $\gamma$ $=\left\{2.791 \times 10^{-4}, 2.933 \times 10^{-2}, 3.163 \times 10^{-1}\right\}$ and $e \phi_{0}(0) / T=\left\{9.247 \times 10^{1}, 2.321 \times 10^{1}, 6.155\right\}$, respectively, for $a=1.125 \mathrm{~cm}$, and $\gamma=\left\{1.771 \times 10^{-8}, 2.791\right.$ $\left.\times 10^{-4}, 2.933 \times 10^{-2}\right\}, e \phi_{0}(0) / T=\left\{2.361 \times 10^{2}, 5.820 \times 10^{1}, 1.426 \times 10^{1}\right\}$ for $a=2.25 \mathrm{~cm}$. The parameters $a=1.125 \mathrm{~cm}$ and $\lambda_{D} / R=0.05$ are typical for the Eltrap experiment, characterized by $n_{*}=1 . \times 10^{7} \mathrm{~cm}^{-3}, T=1 \mathrm{eV}, \lambda_{D}=0.24 \mathrm{~cm}, R=4.5 \mathrm{~cm}$.

$$
\phi_{1}^{(1 *)}(r)=\phi_{1}^{(1)}(r)-R \frac{d \phi_{0}(r)}{d r}
$$

of the electric potential perturbation $\phi_{1}^{*}(r, \theta, z)$ $=\phi_{1}^{(1 *)}(r)(\alpha z / R) \cos \theta$ is drawn in Fig. 3(c). The comparison of Figs. 3(c) and 3(a) demonstrates that the second term in Eq. (82) is really dominating, but it is emphasized again that this fact is due to the magnetic field displacement, while the electric field perturbation that governs the motion of the particles in the plasma is entirely due to the first term. This fact, in turn, supports once again the statement that a selfconsistent theory of non-neutral plasma transport is most easily formulated in flux coordinates.
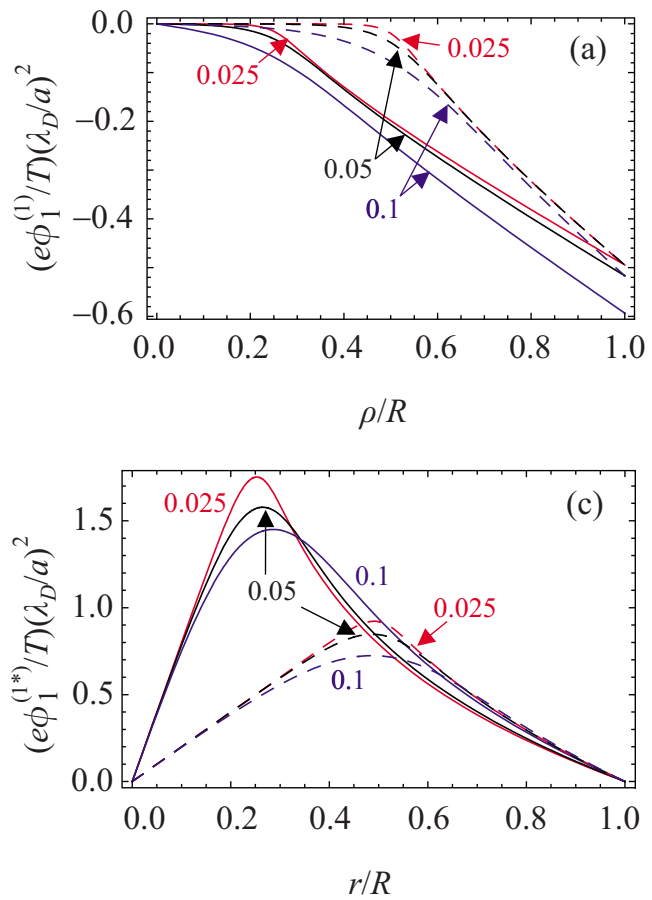

FIG. 5. (Color online) Perturbed potentials $\phi_{1}^{(1)}(\rho), \phi_{1}^{(2)}(\rho)$ in flux coordinates (graphs a and b, respectively) and the same profiles $\phi_{1}^{(1 *)}(r), \phi_{1}^{(2 *)}(r)$ in device coordinates (graphs $\mathrm{c}$ and d) for a non-neutral plasma column with a smooth density profile. The correspondence of the parameters to solid and dashed curves of different colors is the same as in Fig. 4.
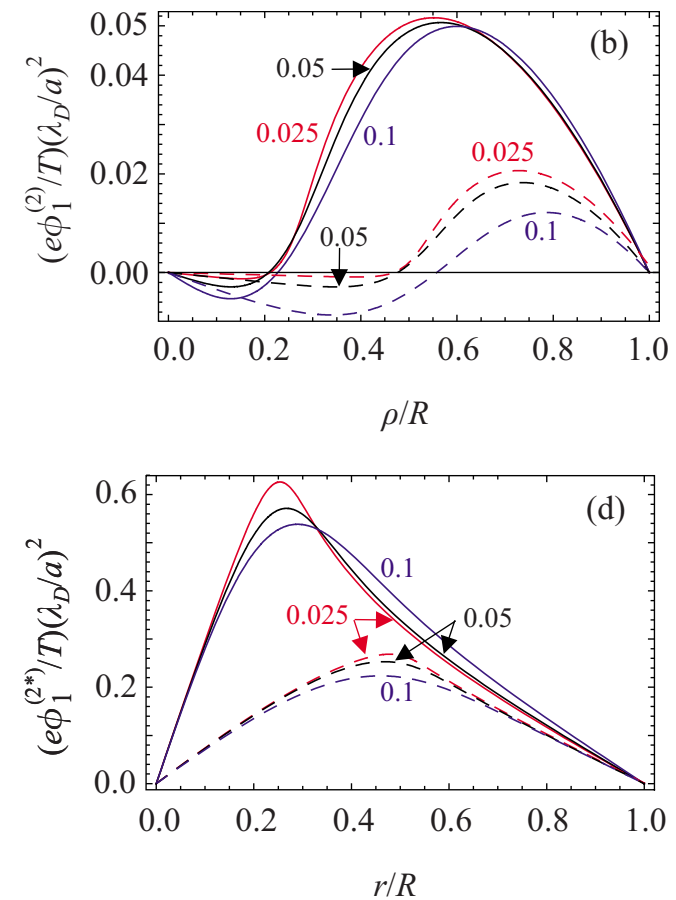

\section{B. Thermal equilibrium density profile}

In the state of global thermal equilibrium, characterized by a rigid rotation of the plasma column around its own axis, the unperturbed density and electric potential profiles have the form shown in Fig. 4 for a set of parameters relevant to the Eltrap experiment. ${ }^{28}$ The profiles are characterized by the parameter

$$
\gamma=-2 \omega(\Omega+\omega) / \omega_{p}^{2}-1,
$$

which effectively determines the radius of the plasma column for given values of the angular rotation frequency, $\omega$, the cyclotron frequency, $\Omega=e B_{*} / m c$, and the plasma fre- 

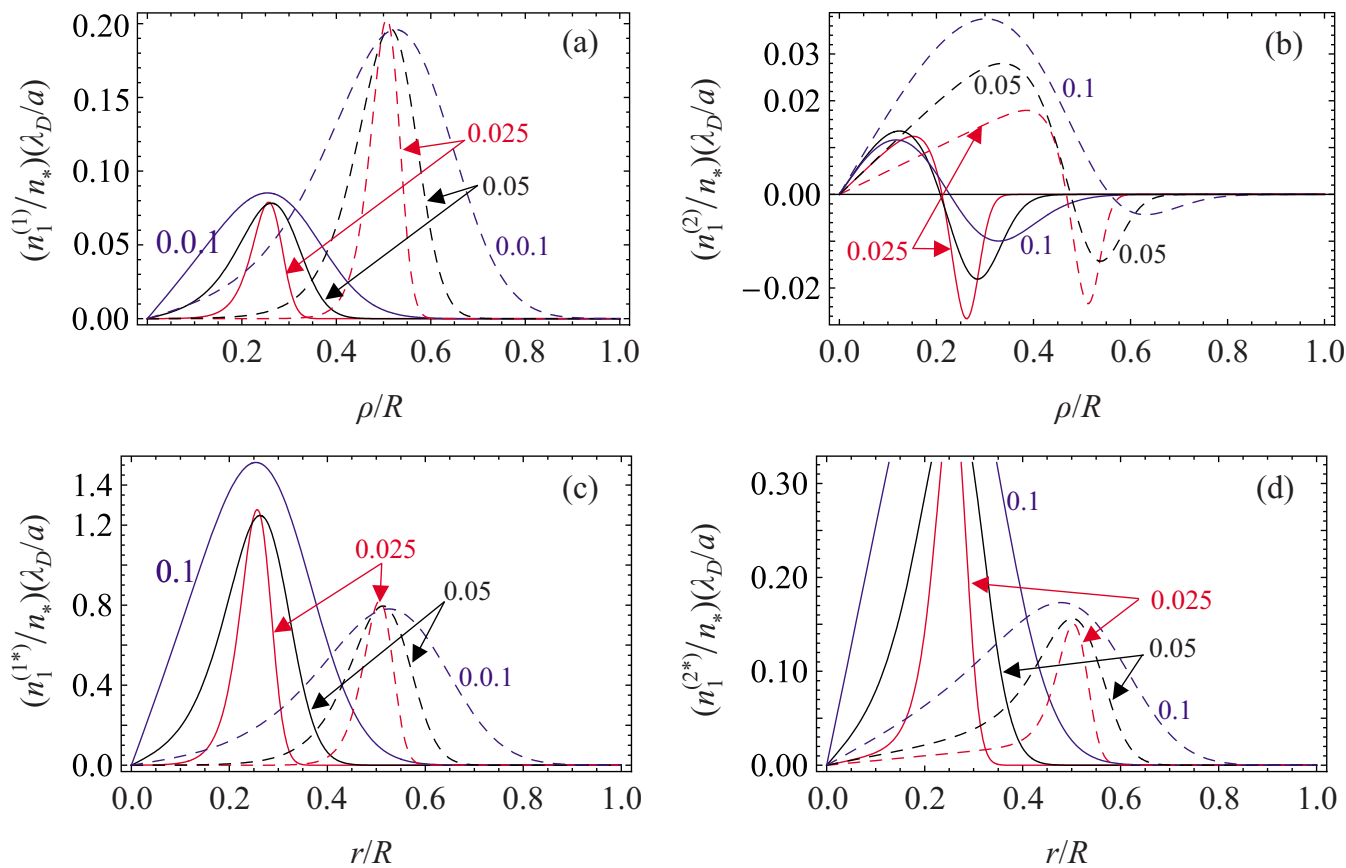

FIG. 6. (Color online) Radial profiles $n_{1}^{(1)}(\rho)(\mathrm{a}), n_{1}^{(2)}(\rho)(\mathrm{b})$ of the perturbed plasma density in flux coordinates and the same profiles $n_{1}^{1 *}(r)(\mathrm{c}), n_{1}^{2 *}(r)(\mathrm{d})$ in device coordinates. The parameters are indicated in Fig. 4.

quency at the column axis, $\omega_{p}=\sqrt{4 \pi e^{2} n_{*} / m}$. Localized density profiles exist if $\gamma>0$, and the plasma radius can be roughly evaluated as $a \sim \lambda_{D} \ln (1 / \gamma)$. The details of the global thermal equilibrium calculations relevant to the problem under consideration can be found in Refs. 14, 27, and 29. Note that in the state of global thermodynamic equilibrium the density needs not to be constant as it is incorrectly stated in Ref. 15.

The radial profiles for the perturbed electric potential, computed from the linearized equations (74) and (75), are shown in Figs. 5(a) and 5(c). Unessential differences are found with respect to the profiles in Figs. 3(a) and 3(c), showing many common features with the analytically solvable model of a plasma with a stepwise density profile.

The perturbation of the plasma density along a field line follows the profile of the electric potential since

$$
n_{1}(\rho, \vartheta, \zeta)=-n_{0}(\rho) \frac{e \phi_{1}(\rho, \vartheta, \zeta)}{T} .
$$

Similar to Eq. (81), for the perturbation of the plasma density expressed in device coordinates one has

$$
n_{1}^{*}(r, \theta, z)=-n_{0}^{\prime}(r) \rho_{1}(r, \theta, z)+n_{1}(r, \theta, z) .
$$

Analogously to $\phi_{1}^{*}(r, \theta, z)$, the function $n_{1}^{*}(r, \theta, z)$ represents the behavior of the perturbed density along a straight line (labeled by fixed values of $r$ and $\theta$ ) parallel to the device axis.

The radial profiles

$$
n_{1}^{(1)}(\rho)=-n_{0}(\rho) \frac{e \phi_{1}^{(1)}(\rho)}{T}
$$

and

$$
n_{1}^{(1 *)}(r)=n_{1}^{(1)}(r)-R \frac{d n_{0}(r)}{d r}
$$

are plotted in Figs. 6(a) and 6(c), respectively. The relation between $n_{1}^{(1)}(\rho)$ and $n_{1}(\rho, \vartheta, \zeta)$ is the same as that between $\phi_{1}^{(1)}(\rho)$ and $\phi_{1}(\rho, \vartheta, \zeta)$; see Eq. (73). The edge peaks of $n_{1}$ become narrower when the plasma temperature is decreasing ( $\lambda_{D}$ becomes smaller). The positive sign of the peaks in Fig. 6 means that the plasma is denser in the regions of the column closer to the conducting walls. In other words the plasma is attracted to the walls, and it tends to be closer to the wall than what would be expected from simply following the magnetic field lines. A similar effect was first noted in Ref. 30.

In contrast to the case of a magnetic squeeze,${ }^{14}$ the radial profile of the perturbation of the electric potential $\phi_{1}^{(1)}(\rho)$ exhibits no sign reversal but the perturbation $\phi_{1}(\rho, \vartheta, \zeta)$ per se changes its sign on the opposite side of the column, both in the $z$ - and in the $\theta$-direction, as it is clearly shown by the contour plots of the plasma density cross sections in Fig. 7. The top row shows the contour plots of the total plasma density in flux coordinates, the middle row the same contours in device coordinates, and the bottom row the isocontours of the perturbed density $n_{1}$ in flux coordinates. For reasonably small values of the tilt angle $\alpha$, the deformation of the contours of constant density from the circular shape is not visible in the scale of the figure.

Figure 8 illustrates the structure of the density perturbations with the use of $3 \mathrm{D}$ isocontours in flux coordinates. The perturbation has opposite signs at the ends of the plasma column and after a half turn around the plasma axis. As a 


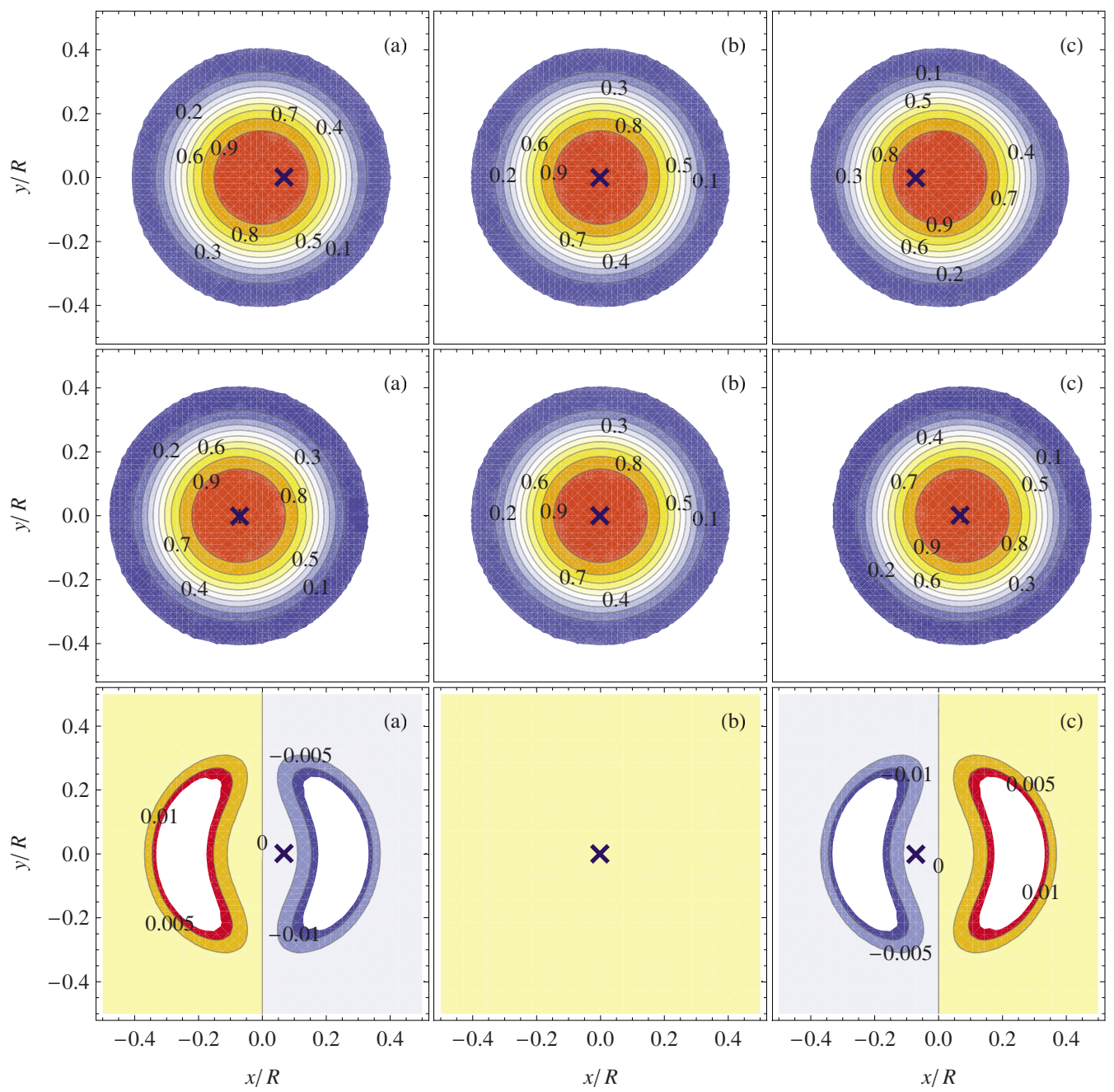

FIG. 7. (Color online) Uniform magnetic tilt. Contours of the total plasma density in three cross sections of the column: $z=-4 R$ (a), $z=0$ (b), $z=+4 R$ (c) in flux coordinates (top row) and in device coordinates (middle row), and contours of the plasma density perturbation in flux coordinates (bottom row); $\alpha$ $=1^{\circ}, a / R=0.25, \lambda_{D} / R=0.05$. The minimum density level shown for the total plasma density corresponds to $n / n_{*}=0.005$. The other parameters are indicated in Fig. 4. The cross indicates the position of the device axis in the top and the bottom row, and the position of the plasma column axis in the middle row.

result, the linear density of the plasma column remains approximately constant along the column; this fact has been first noted in numerical simulations of the plasma equilibrium in a weakly tilted magnetic field in Ref. 9.

Similar to Fig. 7, Fig. 9 shows contour plots (in flux coordinates) of the total electric potential $\phi_{0}+\phi_{1}$ (top row) and of the perturbed part $\phi_{1}$ alone in the same cross sections (bottom row).

The maximum perturbation of the plasma density near the plasma edge is estimated as

$$
\frac{n_{1}}{n_{*}} \sim \frac{a^{2}}{2 R \lambda_{D}} \frac{\alpha \zeta}{R} \cos \vartheta
$$

as it can be deduced from Fig. 6. This estimate agrees very well with that of the perturbed potential near the plasma edge Eq. (66), using the solution Eqs. (80a)-(80c) for a stepwise density profile and the relation (83) between $n_{1}$ and $\phi_{1}$.

The estimate Eq. (87) allows us to evaluate the range of validity of the weak perturbation approximation adopted in

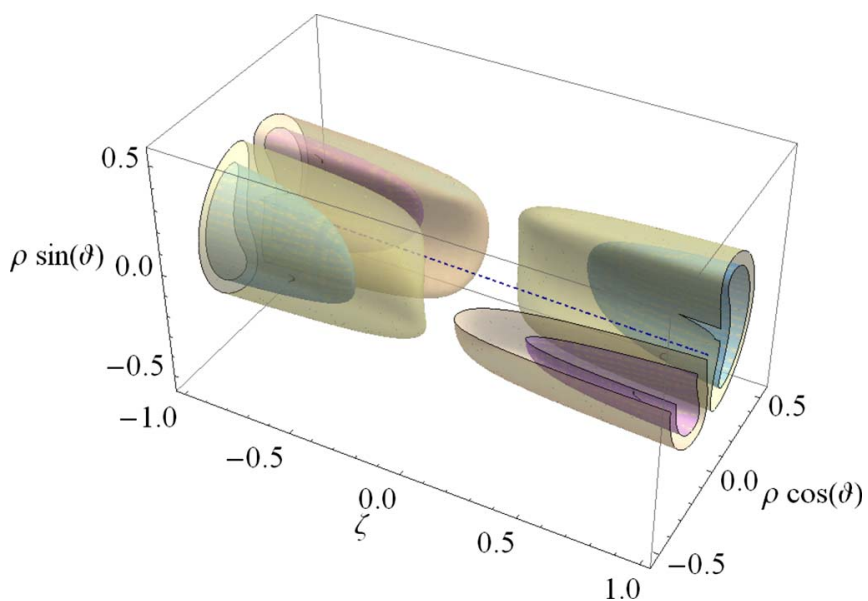

FIG. 8. (Color online) Uniform magnetic tilt. 3D isocontours of the plasma density perturbation $n_{1}(\rho, \vartheta, \zeta)$ in flux coordinates at the levels -0.005 (purple inner surfaces), +0.005 (blue inner surfaces), and \pm 0.001 (lighter outer surfaces); $\alpha=2^{\circ}, \lambda_{D} / R=0.05, a / R=0.25$. The front top quarter of the picture is removed for illustrative purposes. The axis coordinates are normalized over $R$. 


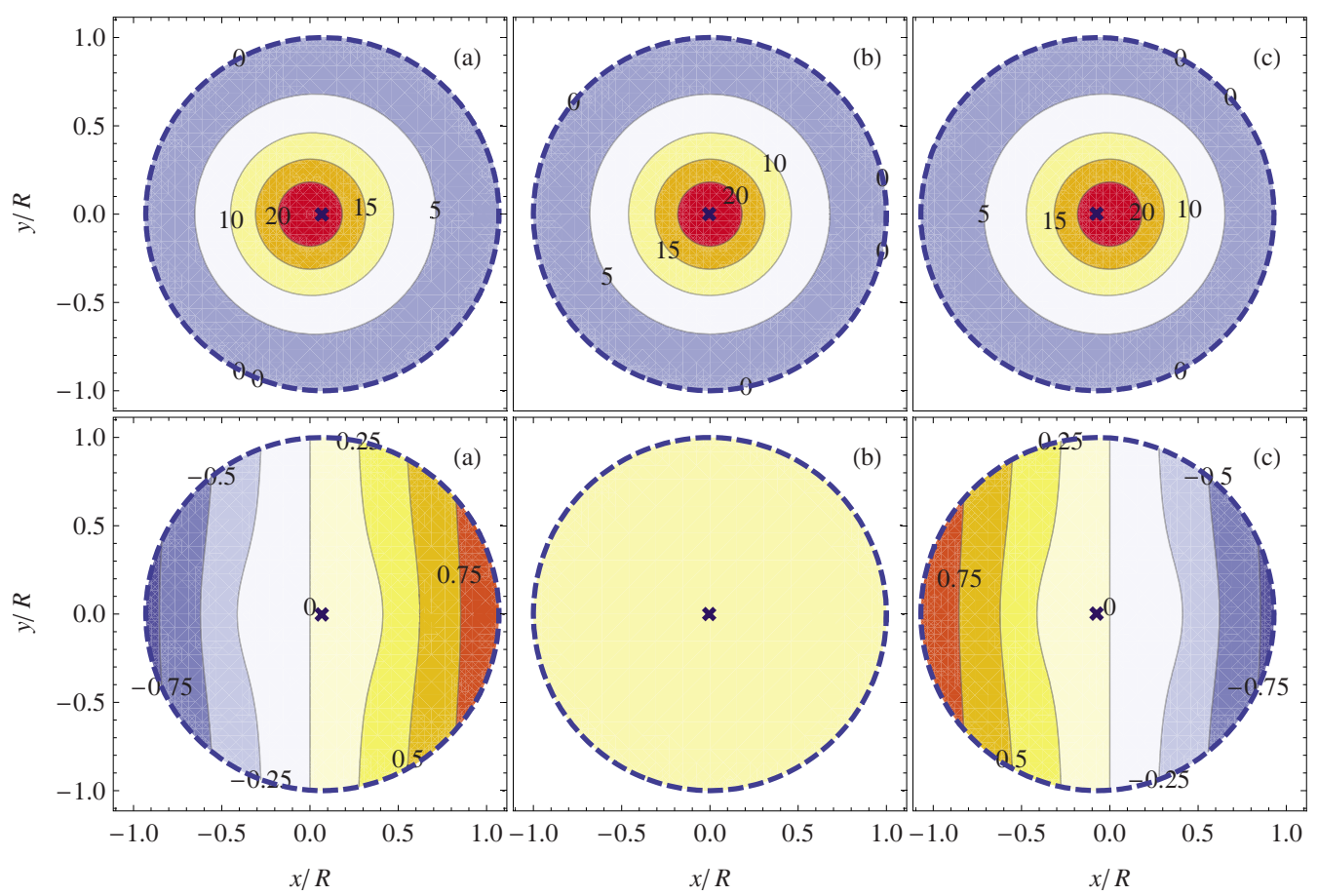

FIG. 9. (Color online) Uniform magnetic tilt. Contours of the electric potential in flux coordinates for three cross sections of the column: $z=-4 R$ (a), $z=0$ (b), $z=+4 R$ (c). Top row: total potential $\phi_{0}+\phi_{1}$; bottom row: perturbed potential $\phi_{1} ; \alpha=1^{\circ}, \lambda_{D} / R=0.05, a / R=0.25$. The dashed circle indicates the conducting wall.

this paper. One expects that the theory is valid if $n_{1}$ is small in comparison with $n$ on the same field line. In other words, the condition

$$
\frac{\alpha L}{\lambda_{D}} \frac{a^{2}}{R^{2}} \ll 1
$$

must hold. From Eq. (80a) one can deduce that this inequality means $e \phi_{1} / T \ll 1$.

It is worth noting that Eq. (86) requires an even stronger inequality to be valid. Indeed, the use of the expansion $n_{0}\left(r-\rho_{1}\right) \approx n_{0}(r)-\rho_{1} d n_{0} / d r$ for its derivation implies that $\rho_{1} d n_{0} / d r \ll n_{0}(r)$. Denoting the width of the plasma density edge layer by $\Delta$, the latter inequality is equivalent to the condition

$$
\frac{\alpha L}{\Delta} \ll 1
$$

which is more stringent than the relation (88) if $\Delta$ $<(R / a)^{2} \lambda_{D}$. It remains more stringent even if the physically reasonable estimate $\Delta \sim \lambda_{D}$ for the edge layer width is adopted. However, the violation of the inequality (89) means simply that using the function (86) to represent the density perturbation in device coordinates becomes inadequate. In that case it is still possible to keep $n_{0}\left(r-\rho_{1}\right)$ in an unexpanded form, at least in the vicinity of the edge layer. No such troubles occur when flux coordinates are used.

A similar validity analysis for Eq. (82) gives the relation

$$
\frac{\alpha L}{a} \ll \ln \frac{R}{a},
$$

which turns out to be less stringent than all conditions listed above. This means that the function $\phi_{1}^{(1 *)}(r)$ can be used to represent the perturbation of the electric potential in device coordinates without any restrictions, as long as the condition (88) holds.

In practice, all calculations have been performed here in flux coordinates. To plot the results in device coordinates an accurate transformation has to used, as described in Appendix B.

\section{REVERSIBLE MAGNETIC TILT}

In this section the case of a magnetic tilt localized in the central part of the confinement region of the plasma is considered. This kind of tilt perturbation can be easily introduced in a magnetic system composed of a set of equidistant equal coils. If one of the coils is slightly tilted around one of its diameters, it gives rise to a dipole perturbation of the magnetic field localized within a range of approximately four radii of the coil. Denoting with $b$ the radius of the coils and with $\ell$ the distance between neighboring coils, the longitudinal magnetic field on the device axis is

$$
B_{0}(z)=\frac{B_{*} b^{2} \ell}{2\left(b^{2}+z^{2}\right)^{3 / 2}}
$$

for a coil located at the plane $z=0$. The sum of many such fields, centered at integer multiples of $\ell$ along $z$, actually 


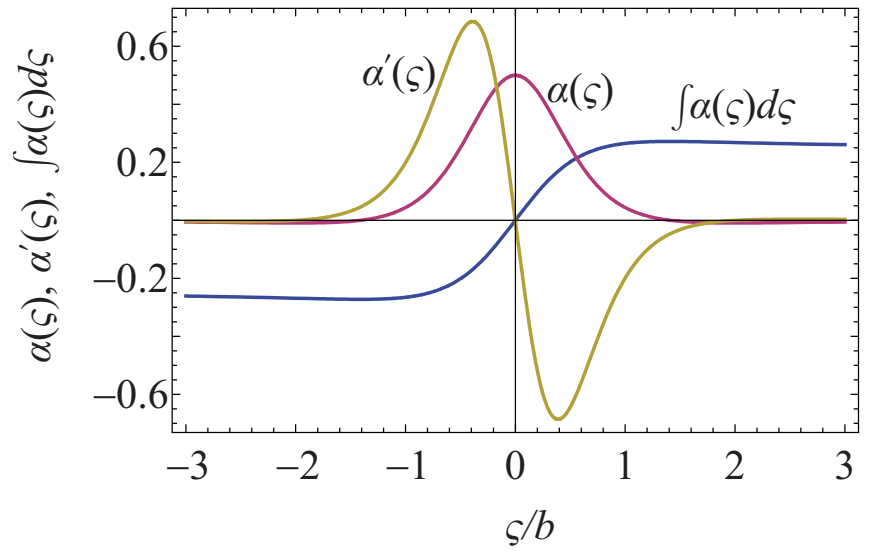

FIG. 10. (Color online) Tilted magnetic field produced by a slightly rotated single coil. The tilt angle $\alpha(\zeta)$ in normalized over $\epsilon l / b$, the derivative $\alpha^{\prime}(\zeta)$ over $\epsilon l / b^{2}$, and the integral $\int \alpha d \zeta$ over $\epsilon l$. The maximum tilt angle $\alpha_{\max }$ $=\epsilon l / 2 b$ is significantly smaller than the inclination angle $\epsilon$ of the coil. The shape of the plasma axis (i.e., the displacement $x$ of the plasma column center in device coordinates) is given by the equation $x=\int \alpha d \zeta$, and the maximum displacement $\epsilon \ell / 4$ is achieved at a distance from the coil approximately equal to its radius $b$.

gives a uniform magnetic field $B_{*}$ provided that $b / \ell$ is sufficiently large. If the selected coil is rotated by a small angle $\epsilon$ around the $y$-axis, it gives rise to the dipole component of the magnetic field

$$
B_{1 x}(z)=\epsilon\left[B_{0}(z)+\frac{1}{2} z B_{0}^{\prime}(z)\right] .
$$

A straightforward calculation then gives the tilt angle $\alpha$ $=B_{1 x} / B_{*}$ as a function of the coordinate $z$,

$$
\alpha(z)=\epsilon \frac{b^{2} \ell\left(2 b^{2}-z^{2}\right)}{4\left(b^{2}+z^{2}\right)^{5 / 2}} .
$$

The tilt angle $\alpha(z)$, its derivative $\alpha^{\prime}(z)$ and the integral $\int \alpha d z$ are plotted in Fig. 10. The tilt angle vanishes at a distance $|z|=2 b$ from the coil and it is extremely small at large distances. Within the range $|z|<2 b$ the tilt angle can be roughly estimated as $\alpha \sim \epsilon l / b \ll \epsilon$, reaching the maximum value exactly under the coil at $z=0$.

For a nonuniform tilt the Poisson equation (58) for the perturbed part of the electric potential takes the form

$$
\begin{aligned}
\frac{1}{\rho} \frac{\partial}{\partial \rho} \rho & \frac{\partial}{\partial \rho} \phi_{1}+\frac{1}{\rho^{2}} \frac{\partial^{2}}{\partial \vartheta^{2}} \phi_{1}+\frac{\partial^{2}}{\partial \zeta^{2}} \phi_{1} \\
= & -\alpha^{\prime}\left[\frac{3}{2} \rho \frac{\partial^{2} \phi_{0}}{\partial \rho^{2}}+\frac{1}{2} \frac{\partial \phi_{0}}{\partial \rho}+2 \rho^{\frac{\partial^{2} \phi_{0}}{\partial \zeta^{2}}}\right] \cos \vartheta \\
& +4 \pi e\left[N_{0} \frac{e \phi_{1}}{T}-N_{1}\right] e^{-e \phi_{0} T} .
\end{aligned}
$$

The first of Eqs. (56) reads

$$
\rho_{1}=\left[\int_{\zeta_{0}}^{\zeta} \alpha(\zeta) d \zeta-\frac{3}{8} \alpha^{\prime}(\zeta) \rho^{2}\right] \cos \vartheta,
$$

so that the boundary condition $(59 \mathrm{~b})$ is given by
$\phi_{1}(R, \vartheta, \zeta)=\left[\int_{\zeta_{0}}^{\zeta} \alpha(\zeta) d \zeta-\frac{3}{8} \alpha^{\prime}(\zeta) R^{2}\right] \frac{d \phi_{0}(R)}{d R} \cos \vartheta$

For long-thin perturbations, such as those produced by a tilted coil with $b \gg(a, R)$, the last term in the left-hand side of Eq. (94), $\partial^{2} \phi_{1} / \partial \zeta^{2}$, as well as $\alpha^{\prime} \partial^{2} \phi_{0} / \partial \zeta^{2}$ in the righthand side can be dropped. Then, the analysis of Eqs. (94) and (96) with the same method used in Sec. VIII for the case of a uniform tilt, suggests that the perturbed potential can be sought in the form ${ }^{31}$

$$
\begin{aligned}
\phi_{1}(\rho, \zeta, \vartheta)= & {\left[\frac{1}{R} \int_{\zeta}^{\zeta} \alpha(\zeta) d \zeta-\frac{3}{8} R \alpha^{\prime}(\zeta)\right] \phi_{1}^{(1)}(\rho) \cos \vartheta } \\
& +\left[R \alpha^{\prime}(\zeta)\right] \phi_{1}^{(2)}(\rho) \cos \vartheta,
\end{aligned}
$$

where the factors $R^{-1}$ and $R$ have been added in front of $\int \alpha d \zeta$ and $\alpha^{\prime}$, respectively, in order to have the same dimensionality for the functions $\phi_{1}^{(1)}(\rho), \phi_{1}^{(2)}(\rho)$, and the potential $\phi_{1}(\rho, \zeta, \vartheta)$.

In Eq. (97) the contribution to $N_{1}$ of the term proportional to $\int_{\zeta_{0}}^{\zeta} \alpha d \zeta$ can be eliminated by a proper choice of the origin of the $\zeta$ axis, i.e., by a proper choice of $\zeta_{0}$. As in Sec. VIII, it is possible to set $\zeta_{0}=0$ without any loss of generality. The contribution to $N_{1}$ of the second terms, proportional to $\alpha^{\prime}$, is also zero provided that: (1) the perturbation of the magnetic field is localized within the central part of the plasma column where $\phi_{0}=\phi_{0}(\rho)$; and (2) the tilt is reversible, i.e., $\alpha(\zeta)$ vanishes at both ends of the perturbation region. The latter condition is evidently valid for the tilt Eq. (93) produced by a single inclined coil. Limiting the analysis to this particular case, it is assumed that $N_{1}=0$.

Due to the linearity of the Poisson equation (94) and the boundary condition (96), the radial functions $\phi_{1}^{(1)}(\rho)$ and $\phi_{1}^{(2)}(\rho)$ can be determined independently. The function $\phi_{1}^{(1)}(\rho)$ has been actually found in Sec. VIII, while the function $\phi_{1}^{(2)}(\rho)$ is the solution of Eq. (94), where the term containing $N_{1}$ and $\partial^{2}(\ldots) / \partial \zeta^{2}$ are dropped,

$$
\begin{aligned}
\frac{1}{\rho} \frac{d}{d \rho} \rho \frac{d}{d \rho} \phi_{1}^{(2)}-\frac{1}{\rho^{2}} \phi_{1}^{(2)}= & -\frac{1}{R}\left[\frac{3}{2} \rho \frac{\partial^{2} \phi_{0}}{\partial \rho^{2}}+\frac{1}{2} \frac{\partial \phi_{0}}{\partial \rho}\right] \\
& +\frac{4 \pi e^{2} n_{0}}{T} \phi_{1}^{(2)},
\end{aligned}
$$

and with the boundary condition

$$
\phi_{1}^{(2)}(R)=0
$$

since $\phi_{1}^{(1)}$ identically satisfies the boundary condition (96).

Here in the following, the solutions for a stepwise density profile and for the density profile corresponding the a state of global thermal equilibrium are determined. 


\section{A. Stepwise density profile}

For the stepwise density profile (77) the problem specified by Eqs. (97) and (98) can be readily solved, although the result turns out to be rather lengthy,

$$
\frac{e \phi_{1}^{(2)}}{T}=-\frac{\rho}{R}+\frac{I_{1}\left(\rho / \lambda_{D}\right)\left\{R^{2} a^{2}[2 \ln (R / a)-1]+a^{4}+8 R^{2} \lambda_{D}^{2}\right\}}{4 \lambda_{D} R\left[\left(R^{2}-a^{2}\right) I_{0}\left(a / \lambda_{D}\right)+2 a \lambda_{D} I_{1}\left(a / \lambda_{D}\right)\right]}
$$

if $0 \leqslant \rho \leqslant a$, and

$$
\begin{aligned}
\frac{e \phi_{1}^{(2)}}{T}= & \frac{I_{0}\left(a / \lambda_{D}\right)\left\{\left(R^{2}-a^{2}\right) \rho^{2} \ln (R / \rho)-\left(R^{2}-\rho^{2}\right)\left[a^{2} \ln (R / a)+4 \lambda_{D}^{2}\right]\right\} a^{2}}{4 R \lambda_{D}^{2} \rho\left[\left(R^{2}-a^{2}\right) I_{0}\left(a / \lambda_{D}\right)+2 a \lambda_{D} I_{1}\left(a / \lambda_{D}\right)\right]} \\
& +\frac{I_{1}\left(a / \lambda_{D}\right)\left\{2 a^{2} \rho^{2} \ln (R / \rho)+\left(R^{2}-\rho^{2}\right)\left[2 a^{2} \ln (R / a)-a^{2}+8 \lambda_{D}^{2}\right]\right\} a}{4 R \lambda_{D} \rho\left[\left(R^{2}-a^{2}\right) I_{0}\left(a / \lambda_{D}\right)+2 a \lambda_{D} I_{1}\left(a / \lambda_{D}\right)\right]}
\end{aligned}
$$

if $a \leqslant \rho \leqslant R$. The function $\phi_{1}^{(2)}$ is drawn in Fig. 3(b). In the region outside of the plasma column, the function $\phi_{1}^{(2)}$ turns out be numerically smaller than $\phi_{1}^{(1)}$ by approximately an order of magnitude. However it cannot be neglected in the region inside the plasma, especially if the plasma is cold, even though in the complete solution (96) it is multiplied by a smaller factor than $\phi_{1}^{(1)}$.

In the limit of a cold plasma, $\lambda_{D} \ll a$, the formulas (100a) and (100b) simplify to

$$
\begin{aligned}
\frac{e \phi_{1}^{(2)}}{T} \approx & \frac{\left(R^{2}-\rho^{2}\right)\left[2 R^{2} \ln (R / a)-R^{2}+a^{2}\right] a^{3}}{4 R\left(R^{2}-a^{2}\right)^{2} \lambda_{D} \rho} \\
& +\frac{\left[\left(R^{2}-\rho^{2}\right) a^{2} \ln (R / a)-\left(R^{2}-a^{2}\right) \rho^{2} \ln (R / \rho)\right] a^{2}}{4 R\left(a^{2}-R^{2}\right) \lambda_{D}^{2} \rho},
\end{aligned}
$$

outside the plasma column, and

$$
\frac{e \phi_{1}^{(2)}}{T} \approx-\frac{a^{2}\left[2 R^{2} \ln (R / a)-R^{2}+a^{2}\right]}{4\left(R^{2}-a^{2}\right) R \lambda_{D}} \sqrt{\frac{a}{\rho}} \exp \left(\frac{\rho-a}{\lambda_{D}}\right),
$$

inside it, close to the plasma edge, $\lambda_{D} \ll \rho \leqslant a$. Within an edge layer of a few Debye lengths, both functions $\phi_{1}^{(1)}$ and $\phi_{1}^{(2)}$ have approximately a magnitude of order $a^{2} / R \lambda_{D}$. Although $\phi_{1}^{(2)}$ contains the additional factor $\ln (R / a)$ which can be not so small, it enters the full solution (97) multiplied by the factor $R \alpha^{\prime}$, which is $R^{2} / b^{2}$ times smaller than the factor $\int \alpha d \zeta / R$ in front of $\phi_{1}^{(1)}$. Thus, the contribution of $\phi_{1}^{(2)}$ within the edge layer is also negligible and the rough estimate (66) for the variable magnetic tilt remains still valid [one needs only to take into account that $\epsilon L$ transforms into $\epsilon \ell$ for the field Eq. (92)].

However, the contribution of $\phi_{1}^{(2)}$ close to the plasma column axis is essential since

$$
\frac{e \phi_{1}^{(2)}}{T} \approx-\frac{\rho}{R}
$$

whereas $\phi_{1}^{(1)}$ is exponentially small in the same region, see Eq. (85).

It is interesting to note that the relation

$$
\frac{e \phi_{1}}{T} \approx-\frac{B_{1}}{B}
$$

found in Ref. 14 for a magnetic squeeze inside the plasma column occurs to be valid also for a tilt magnetic field perturbation. Observing that the variation of $B_{1}$ along a field line in the case of a tilt magnetic field is $B_{1} / B \approx \alpha^{\prime} \rho \cos \vartheta$ as follows, e.g., from Eq. (60), it is readily seen that Eq. (101c) leads exactly to the relation (102). This means in particular that the calculations of the fractions of magnetically and potentially trapped particles in Ref. 14 are applicable to the case of a magnetic tilt as well. The only difference is that in the present case these fractions are different for distinct magnetic field lines. It is also important that $B_{1}$ changes sign along a magnetic field line if the tilt is reversible as the plot of $\alpha^{\prime}$ as Fig. 10 illustrates.

\section{B. Thermal equilibrium density profile}

The functions $\phi_{1}^{(1)}(\rho)$ and $\phi_{1}^{(2)}(\rho)$ computed for the smooth density profiles presented in Fig. 4 are shown in Fig. 5. Comparing them with the data of Fig. 3 shows once again that the model of a stepwise density profile provides a reasonable approximation for the evaluation of the perturbed electric potential.

A similar statement is not meaningful for the functions $n_{1}^{(1)}(\rho)$ and $n_{1}^{(2)}(\rho)$, since a stepwise profile exhibits huge density gradients at the plasma edge, whereas in reality the plasma density edge layer could hardly be narrower than a Debye length. This is the reason why these functions were not drawn in Secs. VIII A and IX A. For the smooth density profiles they are depicted in Fig. 6.

In Figs. 5(c) and 6(c) the functions $\phi_{1}^{(*)}(r)$ and $n_{1}^{*}(r)$ are shown. They represent respectively the functions $\phi_{1}^{(1)}(\rho)$ and $n_{1}^{(1)}(\rho)$, transformed to device coordinates. A similar transformation for the functions $\phi_{1}^{(2)}(\rho)$ and $\phi_{1}^{(2)}(\rho)$ reads

$$
\phi_{1}^{(2 *)}=\phi_{1}^{(2)}-\frac{3}{8}\left(1-\frac{r^{2}}{R^{2}}\right) R \frac{d \phi_{0}(r)}{d r},
$$




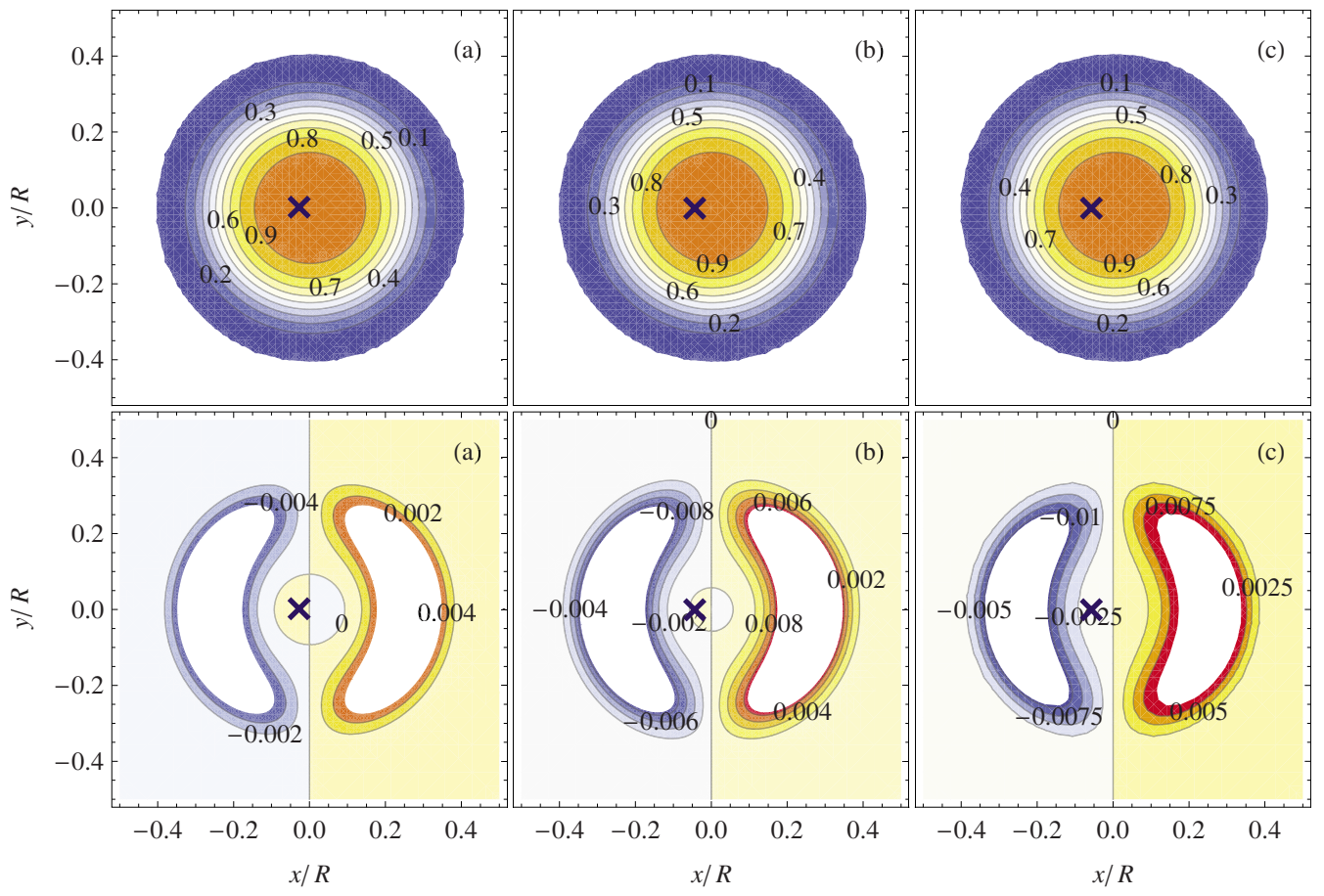

FIG. 11. (Color online) Reversible magnetic tilt. Contours of the plasma density in three cross sections of the column: $z=0.5 R$ (a), $z=R$ (b), $z=2 R$ (c) in flux coordinates. Top row: total plasma density $n_{0}+n_{1}$; bottom row: perturbed plasma density $n_{1}$. The minimum density level shown in the contour plots of the total density corresponds to $n / n_{*}=0.005$. The other parameters are $\epsilon=12^{\circ}, a / R=0.25, \lambda_{D} / R=0.05, b / R=2, b / l=2$. The maximum tilt angle $\alpha(0)=3^{\circ}$ is three times larger than that found in Fig. 7 for a uniformly tilted magnetic field.

$$
n_{1}^{(2 *)}=n_{1}^{(2)}-\frac{3}{8}\left(1-\frac{r^{2}}{R^{2}}\right) R \frac{d n_{0}(r)}{d r} .
$$

Both function vanish for $r=R$.

The contour plots of both the total density $n_{0}+n_{1}$ and of the perturbed density $n_{1}$ alone in several cross sections of the plasma column in the proximity of the tilted coil are drawn in Fig. 11. Figure 12 shows 3D isocontours of the plasma density perturbation $n_{1}$ in flux coordinates. Together with the cross sections shown in Fig. 11 these results demonstrate that

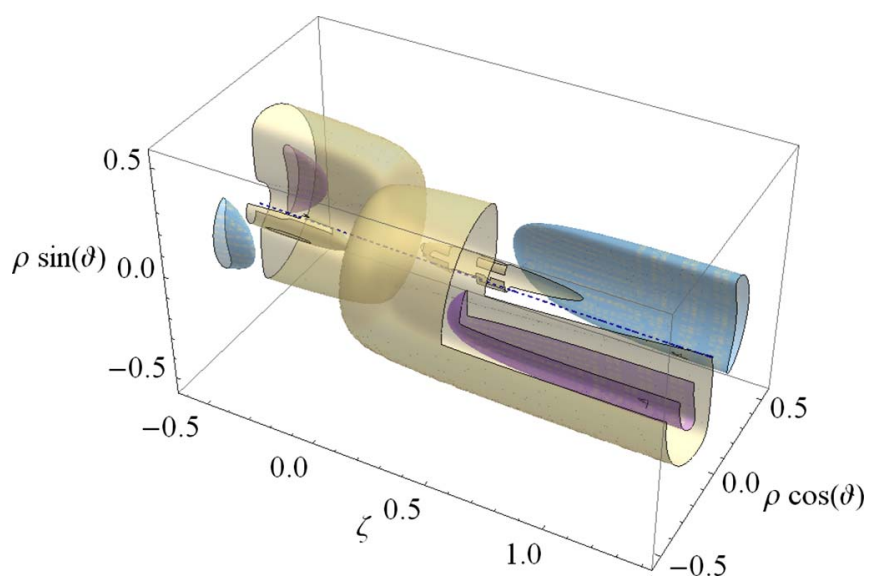

FIG. 12. (Color online) Reversible magnetic tilt produced by a single slightly rotated magnetic coil. 3D isocontours of the plasma density perturbation $n_{1}$ at the levels -0.01 (purple), +0.01 (blue), and -0.0001 (light color); $\epsilon=8^{\circ}$. The other parameters are the same as in Fig. 11. The front top quarter of the picture is removed for illustrative purposes. The axis coordinates are normalized over $R$. the structure of the density perturbation in the case of a reversible tilt is much more complicated as compared to the case of a uniform tilt depicted in Figs. 7 and 8. In the present case, trapped particles appear also close to the axis of the plasma column. Nevertheless, the linear density of the plasma column remains approximately constant along the column. This property follows from the general symmetry of the perturbation which changes sign after an half turn around the plasma axis.

Similar to Fig. 11, Fig. 13 shows contour plots of the total electric potential $\phi_{0}+\phi_{1}$ and of the perturbed part $\phi_{1}$ alone in the cross sections of the plasma column (top and middle row), and the distribution of the electric potential over cylindrical surfaces $\rho=$ const for three different values of $\rho / R$ (bottom row).

The distribution of the perturbed electric potential over the flux surfaces is shown in Fig. 14 for three different values of $\rho$. The results clearly show a different structure for uniform (top row) and reversible tilt (bottom row). In the former case the current streamlines show a similar topology at all radii, although the current density is strongly suppressed close to the plasma column axis. On the contrary, in the case of a reversible tilt the shape of the contours noticeably varies with the radius $\rho$, and the magnitude of the perturbation near the column axis is substantially larger than in the previous case.

\section{PFIRSCH-SCHLÜTER CURRENTS}

In this section the longitudinal plasma currents induced by a magnetic tilt in a non-neutral plasma confined in a 


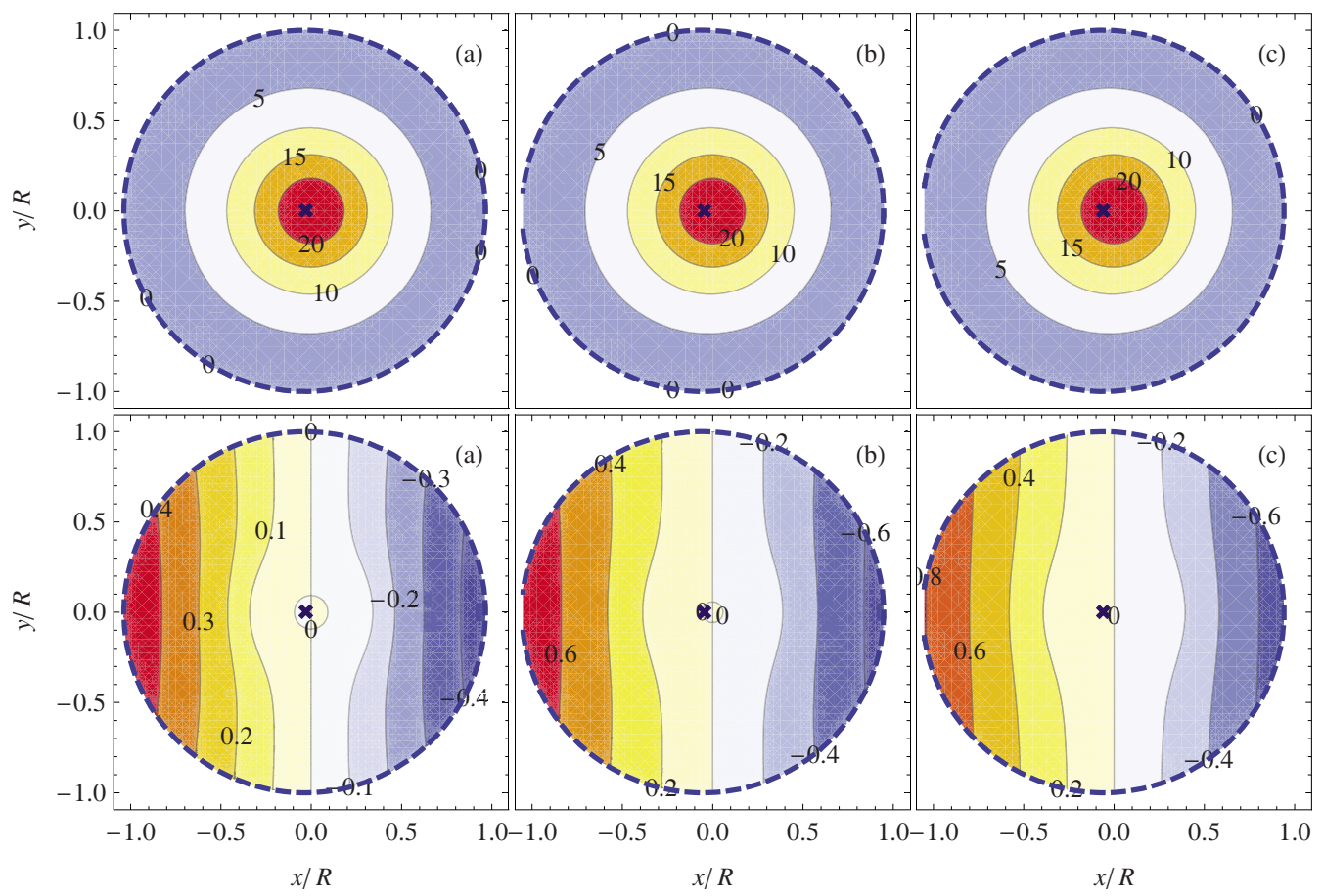

FIG. 13. (Color online) Reversible magnetic tilt. Contours of the electric potential in flux coordinates for three cross sections of the column: $z=0.25 R$ (a), $z=0.5 R$ (b), $z=R$ (c); top row: total potential; bottom row: perturbed potential; $\epsilon=12^{\circ}, \lambda_{D} / R=0.05, a / R=0.25$. The dashed circle indicates the conducting wall.

Malmberg-Penning are calculated. These currents can be thought off as an analog of the Pfirsch-Schlüter currents in tokamaks $^{16}$ or the Stupakov currents in tandem mirrors. ${ }^{3,17}$ However, in the present case these currents have a quite different origin since they appear even in the case of a uniform tilt which does not give rise to any magnetic drift, whereas both the Pfirsch-Schlüter and Stupakov currents originate from magnetic drifts.
The electric current density in a non-neutral plasma is obtained by multiplying Eq. (35) by en:

$$
\mathbf{j}=e n v_{\|} \frac{\mathbf{B}}{B}-c \frac{\partial p}{\partial \rho} \frac{\nabla \rho \times \mathbf{B}}{B^{2}}-c \frac{\partial p}{\partial \vartheta} \frac{\nabla \vartheta \times \mathbf{B}}{B^{2}}
$$

Its contravariant components assume an elegant form,
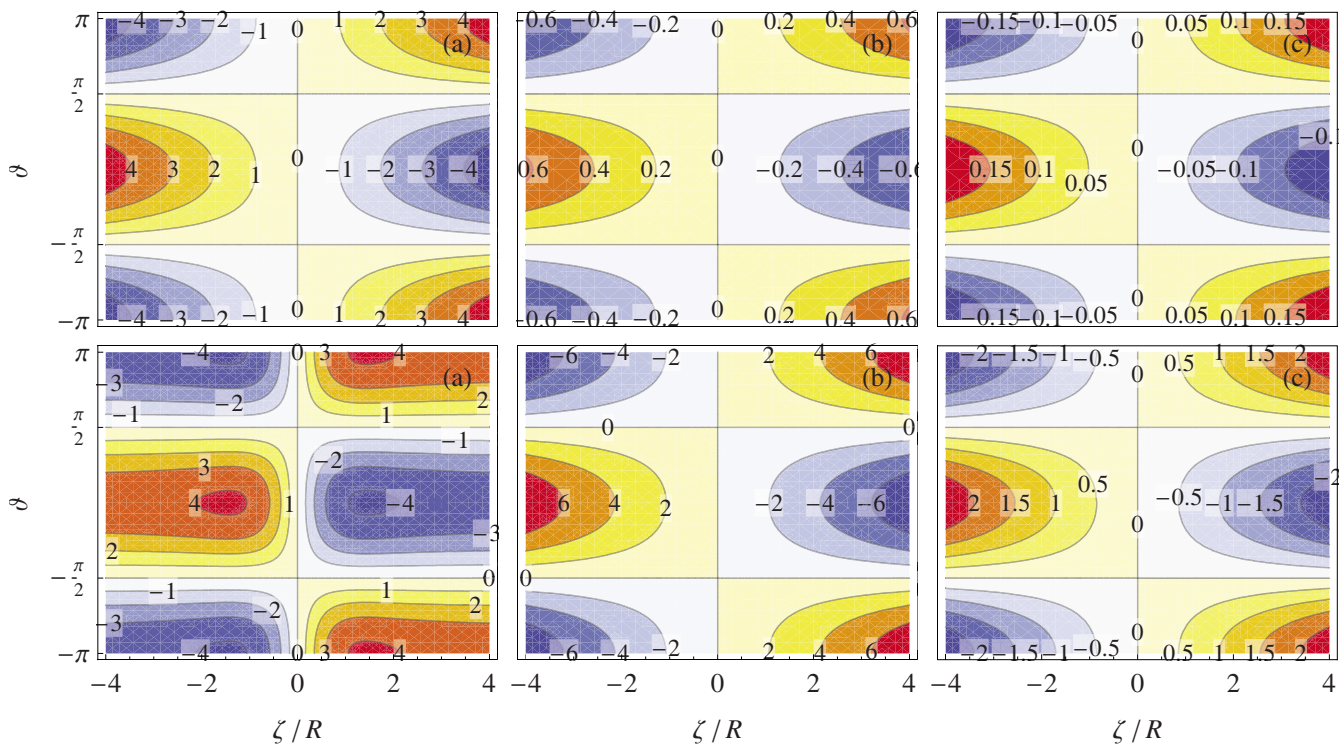

FIG. 14. (Color online) Contours of the perturbed electric potential on flux surfaces with different radii $\rho / R=0.24$ (a), $\rho / R=0.12$ (b), and $\rho / R=0.06$ (d). Top row: uniform magnetic tilt with $\alpha=1^{\circ}$; bottom row: reversible tilt with $\epsilon=12^{\circ}$. The values on the contours indicate the level of the perturbed potential multiplied by a factor of 100. The parameters of uniform and reversible tilt are indicated in Figs. 9 and 11, respectively. 


$$
j^{i}=\left\{-\frac{c}{B_{*} \rho} \frac{\partial p}{\partial \vartheta}, \frac{c}{B_{*} \rho} \frac{\partial p}{\partial \rho}, e n v_{\|} \frac{B}{B_{*}}\right\} .
$$

The longitudinal plasma current $j^{3}=e n v_{\|} B / B_{*}$ is obtained by integrating Eq. (39) over $\zeta$ along a magnetic field line,

$$
\begin{aligned}
j^{3}= & \frac{c B^{2}}{B_{*}} \int_{-\infty}^{\zeta} d \zeta\left\{-\frac{\partial p}{\rho \partial \rho} \frac{\partial}{\partial \vartheta} \frac{1}{B^{2}}+\frac{\partial p}{\partial \vartheta} \frac{\partial}{\rho \partial \rho} \frac{1}{B^{2}}\right. \\
& \left.+\frac{1}{B^{2}} \frac{\partial e \phi}{\partial \vartheta} \frac{\partial}{\rho \partial \rho} \frac{p}{T}-\frac{1}{B^{2}} \frac{\partial e \phi}{\rho \partial \rho} \frac{\partial}{\partial \vartheta} \frac{p}{T}\right\} .
\end{aligned}
$$

As it is explained in Sec. VIII, the first two terms in the right-hand side can be dropped. The last term is zero since $\partial(p / T) / \partial \vartheta=\partial N_{1} / \partial \vartheta$, and $N_{1}=0$ for all examples considered in this paper. Reminding that the derivatives of $p$ over $\rho$ and $\theta$ are taken at fixed $\phi$, the longitudinal current density $j^{3}$ reduces to

$$
j^{3}=\frac{c}{B_{*}} \int_{-\infty}^{\zeta} \frac{\partial e \phi_{1}}{\partial \vartheta} \frac{\partial N_{0}}{\rho \partial \rho} \exp \left(-\frac{e \phi_{0}}{T}\right) d \zeta .
$$

In the case $T=$ const, the derivative $\partial p / \partial \vartheta$ is proportional to $\partial N_{1} / \partial \vartheta$, and, hence, is equal to zero. As a consequence, the radial current density is

$$
j^{1}=-\frac{c}{B_{*} \rho} \frac{\partial p}{\partial \vartheta}=0 .
$$

The azimuthal component of the current density

$$
j^{2}=\frac{c}{B_{*} \rho} \frac{\partial p}{\partial \rho} \approx \frac{c T}{B_{*} \rho} \frac{\partial N_{0}}{\partial \rho} \exp \left(-\frac{e \phi_{0}}{T}\right)\left[1-\frac{e \phi_{1}}{T}\right]
$$

contains both unperturbed and perturbed contributions, the latter being given by the second term in brackets.

Introducing the flux function

$$
\Psi_{1}=\frac{c T}{B_{*}} \frac{\partial N_{0}}{\partial \rho} \int_{-\infty}^{\zeta} \frac{e \phi_{1}}{T} \exp \left(-\frac{e \phi_{0}}{T}\right) d \zeta
$$

one can represent the perturbed part of the current density as

$$
j_{1}^{2}=-\frac{1}{\rho} \frac{\partial \Psi_{1}}{\partial \zeta}, \quad j_{1}^{3}=\frac{1}{\rho} \frac{\partial \Psi_{1}}{\partial \vartheta} .
$$

The contravariant quantities (110) are already of order $\epsilon^{1}$. Therefore $1 / \rho$ can be considered as $1 / \sqrt{g}$ within the desired accuracy. This casts Eq. (110) into the vector form

$$
\mathbf{j}_{1}=\nabla \rho \times \nabla \Psi_{1} .
$$

It follows that the level contours of $\Psi_{1}$ on a surface with given radius $\rho$ are just the current lines.

For the case of a uniform tilt the flux function (109) can be approximated by the expression

$$
\Psi_{1}=\frac{c T}{B_{*}}\left[\frac{d n_{0}}{d \rho}+n_{0} \frac{d}{d \rho} \frac{e \phi_{0}}{T}\right] \frac{e \phi_{1}^{(1)}(\rho)}{T} \alpha \frac{\zeta^{2}-L^{2} / 4}{2 R} \cos \vartheta .
$$

To obtain Eq. (112) it has been assumed that the plasma column has a length $L \gg R$, and that the external electric potential grows sufficiently rapidly at the column ends so that the end regions are very short as compared to the total length
$L$. In addition, the following identity has been used:

$$
\frac{d N_{0}}{d \rho} \exp \left(-\frac{e \phi_{0}}{T}\right)=\frac{d n_{0}}{d \rho}+n_{0} \frac{d}{d \rho} \frac{e \phi_{0}}{T},
$$

following from the definition of $n_{0}(\rho)=N_{0}(\rho) e^{-e \phi_{0} / T}$.

The above calculations can be easily extended to the case of a variable magnetic tilt. The result has the form

$$
\begin{aligned}
\Psi_{1}= & \frac{c T}{B_{*}}\left[\frac{d n_{0}}{d \rho}+n_{0} \frac{d}{d \rho} \frac{e \phi_{0}}{T}\right]\left\{\left[\beta_{1}(\zeta)-\beta_{1}(L / 2)\right] \phi_{1}^{(1)}\right. \\
& \left.+\left[\beta_{2}(\zeta)-\beta_{2}(L / 2)\right] \phi_{1}^{(2)}\right\} \cos \vartheta,
\end{aligned}
$$

where

$$
\begin{aligned}
& \beta_{1}(\zeta)=\int_{0}^{\zeta} d \zeta \int_{0}^{\zeta^{\prime}} d \zeta^{\prime} \alpha\left(\zeta^{\prime}\right) / R^{2}-\frac{3}{8} \alpha(\zeta), \\
& \beta_{2}(\zeta)=\alpha(\zeta) .
\end{aligned}
$$

Examples of the electric current streamlines are drawn in Fig. 15 at different radii. They have the same shape at each radius in the case of a uniform magnetic tilt (top row) but change significantly with $\rho$ in the case of a reversible tilt (bottom row).

For a known $\Psi_{1}$, the current density can be readily found using Eq. (111). For example, the components of $\mathbf{j}$ for a uniform magnetic tilt in the cold plasma limit, $\lambda_{D} \ll a$, can be written explicitly as

$$
\begin{aligned}
j_{\vartheta}= & -\frac{c n_{*} T}{B_{*}} \frac{a^{2}}{R^{2}-a^{2}} \exp \left(\frac{\rho-a}{\lambda_{D}}\right) \sqrt{\frac{a}{\rho}}\left[\frac{1}{2 \lambda_{D}^{2}}+2 \delta\left(\rho^{2}-a^{2}\right)\right] \\
& \times \frac{\alpha \zeta \rho}{\lambda_{D}} \cos \vartheta
\end{aligned}
$$

$$
\begin{aligned}
j_{\zeta}= & -\frac{c n_{*} T}{B_{*}} \frac{a^{2}}{R^{2}-a^{2}} \exp \left(\frac{\rho-a}{\lambda_{D}}\right) \sqrt{\frac{a}{\rho}}\left[\frac{1}{2 \lambda_{D}^{2}}+2 \delta\left(\rho^{2}-a^{2}\right)\right] \\
& \times \alpha \frac{\zeta^{2}-L^{2} / 4}{2 \lambda_{D}} \sin \vartheta
\end{aligned}
$$

where $\delta\left(\rho^{2}-a^{2}\right)=\delta(\rho-a) / 2 \rho$ is a Dirac delta function coming from the differentiation of the stepwise density profile. For a more realistic density profile, the delta function is substituted with a narrow peak with a width of few $\lambda_{D}$ and an amplitude $1 / \lambda_{D} a$ representing a small correction to the first term in brackets. Figure 16 shows radial profiles of the parallel current density $j_{\zeta}$ for the thermal equilibria given in Fig. 4. The profiles are peaked near the column edge, $\rho \sim a$. In the case of a uniform magnetic tilt the maximum value of $j_{\zeta}$ is roughly evaluated from Eq. (114b) as $\left(c T n_{*} / 16 \lambda_{D} B_{*}\right)$ $\times\left(a / \lambda_{D}\right)^{2}\left(\alpha L^{2} / R^{2}\right)$ but actually the peak turns out to be approximately three times smaller; see Fig. 16(a). This discrepancy is explained by the fact that the plasma density is approximately two times lower than $n_{*}$ near the peak of the parallel current.

As $j_{\rho}=0$, the current flows exactly along the surface $\rho$ $=$ const. One can readily check that the particle drift trajectories calculated from the constancy of the longitudinal adia- 


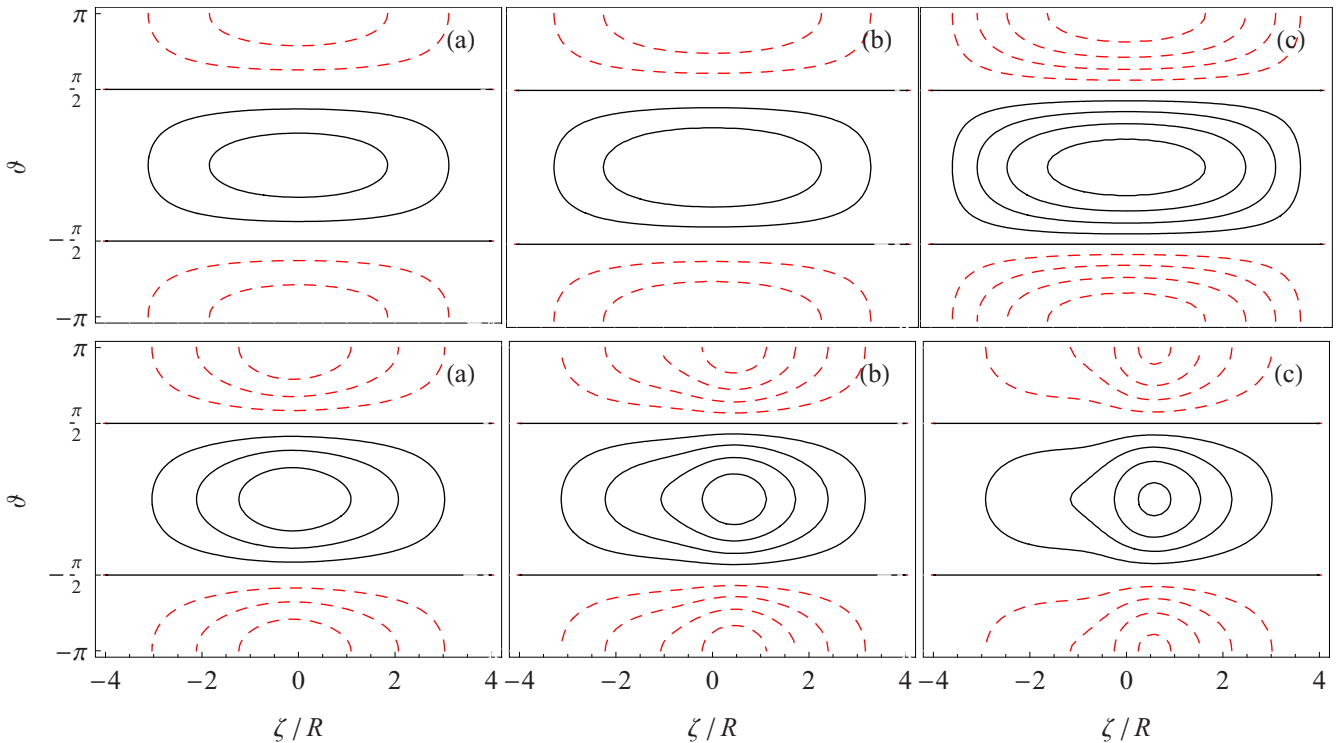

FIG. 15. (Color online) The isocontours of the stream function $\Psi_{1}$ represent the streamlines of the perturbed current density. The maps of the current density in flux coordinates are shown for the radii $\rho / R=0.24$ (a), $\rho / R=0.12$ (b), and $\rho / R=0.06$ (d). Top row: uniform magnetic tilt; bottom row: reversible magnetic tilt produced by a single slightly rotated magnetic coil. Solid and dashed lines correspond to a clockwise and a counterclockwise flowing current, respectively. The parameters of uniform and reversible tilt are indicated in Figs. 9 and 11, respectively.

batic invariant, $J_{\|}=\oint d s v_{\|}$, are also located on $\rho=$ const. This fact leads to an apparent paradox, since for a Maxwellian distribution, which has been implicitly assumed in Sec. IV, the number of particles traveling in opposite directions should be exactly the same in any point and at any instance of time. This means that a longitudinal current should be absent in spite of the above calculations. However, moving in opposite directions the particles are actually radially displaced by a small distance above or below the surface $J_{\|}$ $=$ const, so a parallel current appears due to the radial gradient of the plasma density. A detailed study of the particle motion in the plasma equilibrium configurations computed in this paper will be reported elsewhere.

\section{NUMERICAL PROCEDURE: DISCUSSION}

The analytic treatment developed so far is valid in a long-thin or "paraxial" approximation. In particular, it is assumed that the characteristic axial length of variation of the magnetic field is much longer than its radial scale length. In order to find an equilibrium solution under circumstances in which the asymmetry of the magnetic field is large one has to rely on numerical simulations. This is the case, e.g., of the projects Athena ${ }^{12}$ and Atrap, ${ }^{13}$ aiming to the production and confinement of a weighing amount of antihydrogen atoms. In order to trap these atoms, multipole magnetic fields are added to a conventional Malmberg-Penning trap, whose strengths at the trap wall can be comparable to that of the axial magnetic field.

Before proceeding it is worth noting that the arguments listed in Sec. I in favor of a long term existence of asymmetric equilibria of non-neutral plasmas are based on the smallness of the magnetic field asymmetry. Therefore the existence of strongly asymmetric equilibria might be disputable. At least, it is expected that the plasma edge might not be approximately equipotential as in the case of a weak asymmetry.

The general idea of the numerical procedure can be described in the simpler case in which the temperature $T$ is
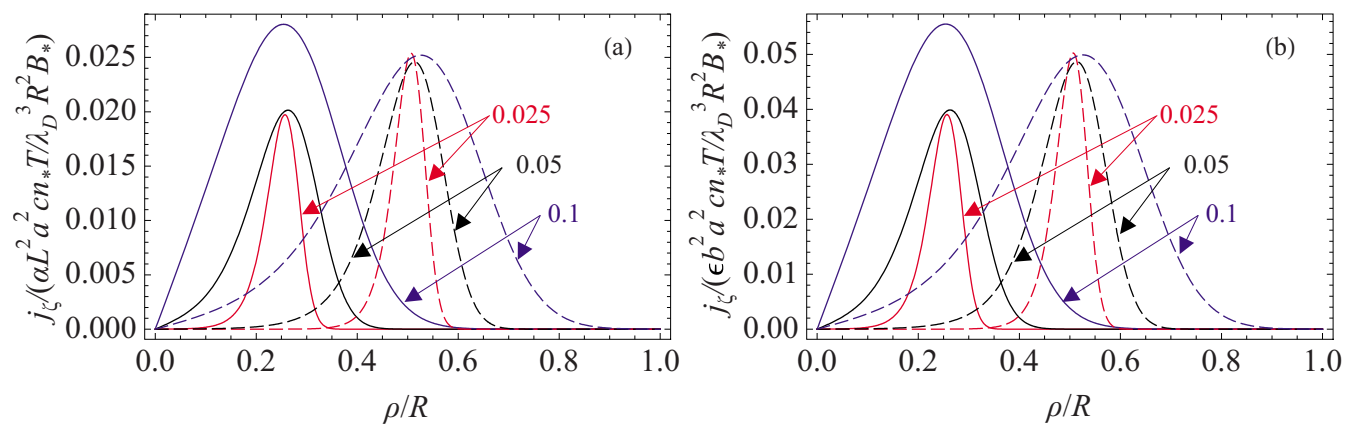

FIG. 16. (Color online) Radial profiles of the parallel current density $j_{\zeta}$ along the ray $\vartheta=\pi / 2, \zeta=0$ for $a / R=0.25$ (solid lines), $a / R=0.5$ (dashed lines), and various values of $\lambda_{D} / R$ (indicated on the plot). (a) Uniform magnetic tilt, $j_{\zeta}$ normalized by $\left(\alpha c n_{*} T / B_{*} \lambda_{D}\right)(L / R)^{2}\left(a / \lambda_{D}\right)^{2}$, (b) reversible magnetic tilt, $j_{\zeta}$ normalized by $\left(\epsilon c n_{*} T / B_{*} \lambda_{D}\right)(b / R)^{2}\left(a / \lambda_{D}\right)^{2}$. The other parameters are indicated in Fig. 4. 
constant (i.e., it does not depend on $\psi$ nor on $\vartheta$ ). First, the Poisson equation (31) can be solved on a 3D mesh for a given density $N(\psi, \vartheta)$. A reasonable initial guess of the density is the solution of the equilibrium equation in the case of an ideally uniform magnetic field described in Sec. VIII B.

The solution for $\phi$ thus determined is then inserted into the solvability condition (43) to find a new solution for $N(\psi, \vartheta)$. The process is then iterated until some convergence criterion is met, e.g., the quantity $\max _{i, j}\left|N_{i j}^{(n+1)}-N_{i j}^{(n)}\right|$ is below a given threshold, where $(i, j)$ are the mesh indices and $(n)$ is the iteration level.

For the case of a magnetic tilt a 3D equilibrium calculation was performed first in Ref. 9, where the perturbed line charge density of the plasma was found to be a linear function of $z$, supporting the analytical results of the present paper. The model assumed a Boltzmann density distribution along the magnetic field and circular symmetry about the center-of-mass in the transverse plane, though the latter assumption was questioned in the same paper. ${ }^{9}$ The Poisson equation was solved with a standard simultaneous overrelaxation algorithm, imposing for the total charge (integrated along the field) to be equal to the experimentally measured value.

An alternative approach is based on 3D PIC simulations. ${ }^{11,32}$ This method allows estimating macroscopic characteristics of the equilibrium states. For example, the critical radius (depending on the order of the multipole and on geometrical and field factors) for the initial plasma column beyond which particles are rapidly lost at the walls could be estimated with simulations of the injection of positrons into the trap. On the other hand, other processes dictated by much slower time scales are more complicated to study with the 3D PIC approach, as it is also stated in Ref. 11. The simulations become too slow to mimic the experimental conditions precisely.

With the aim of modeling the non-neutral plasma equilibrium, the method proposed here could be effectively used instead of the 3D PIC approach.

\section{SUMMARY AND CONCLUSIONS}

In this paper the effect of small asymmetric magnetic field perturbations on the equilibrium of a non-neutral plasma confined in a Malmberg-Penning trap has been analyzed. A constraint has been derived, that selects a class of admissible plasma equilibria in the trap in the presence of a nonuniform and a nonaxisymmetric magnetic field. By analogy with a literally translated Russian expression informally used in the theory of tandem mirrors, this constraint can be called condition of current closure, but in the theory of tokamaks and stellarators a similar condition is indicated as parallel current constraint. ${ }^{4}$ In combination with Poisson's equation this constraint provides a full set of equations for determining self-consistent equilibria of non-neutral plasmas in Malmberg-Penning traps.

Although the parallel current constraint has been applied to analyze plasma equilibria in the case of weak magnetic field perturbations, it can be used for stronger asymmetries such as those required to trap antihydrogen atoms in the
Athena ${ }^{12}$ and Atrap ${ }^{13}$ experiments. A suitable numerical procedure to determine plasma equilibria in these configurations has been briefly outlined.

The effectiveness of the new approach for the determination of non-neutral plasma equilibria has been demonstrated in the case of a weakly tilted magnetic field perturbation. Two examples of analytically solvable equilibria have been given. The simplest example is that of a uniformly tilted magnetic field. The second example, called reversible magnetic tilt, supplies a general method valid for determining non-neutral plasmas equilibria in a weakly nonuniform magnetic field of higher multipolarity such as quadrupole and octupole fields, which will be considered in a future paper. The assumption of ideal cylindricity of the conducting confining chamber implicitly adopted in this paper can be readily relaxed as it was done in Ref. 14, but the effect of the vacuum chamber deformation has not been analyzed in this paper.

It is hoped that completing the analysis of various magnetic and electric potential perturbations and their superpositions on the equilibrium of non-neutral plasmas will open a way for building up a self-consistent theory of non-neutral plasma transport.

\section{ACKNOWLEDGMENTS}

The authors are grateful to be acquainted by Professor T. M. O'Neil with his unpublished derivation of the Poisson equation for a weakly inhomogeneous magnetic field. ${ }^{18}$ Useful discussions with him, Dr. A. Kabantsev, and Dr. T. Akhmetov are gratefully acknowledged.

This work has been started during a visit of I. K. to the Nonneutral Plasma Physics Group of the University of California at San Diego thanks to U.S. Civilian Research and Development Foundation Grant No. RUP1-2631-NO-04. This work was also supported by the Cariplo Foundation and the Landau Network-Centro Volta.

\section{APPENDIX A: EQUATION (48)}

In this section, the proof of the equality (48) is given. Noting that

$$
\frac{\partial \rho_{1}}{\partial \zeta}=-\frac{\partial \zeta_{1}}{\partial \rho},
$$

the derivative of Eq. (48) over $\zeta$ reads

$$
-\nabla^{2} \frac{\partial \zeta_{1}}{\partial \rho}-\frac{1}{\rho^{2}} \frac{\partial \zeta_{1}}{\partial \rho}=\frac{2}{\rho}\left(\frac{\partial^{2} \zeta_{1}}{\partial \zeta^{2}}+\frac{\partial^{2} \zeta_{1}}{\partial \rho^{2}}\right),
$$

since $F$ is independent of $\zeta$. Writing explicitly the operator $\nabla^{2}$ and using the equation $\nabla^{2} \zeta_{1}=0$ in the right-hand side yields

$$
\begin{aligned}
& -\frac{1}{\rho} \frac{\partial}{\partial \rho} \rho \frac{\partial}{\partial \rho} \frac{\partial \zeta_{1}}{\partial \rho}-\frac{1}{\rho^{2}} \frac{\partial^{2}}{\partial \vartheta^{2}} \frac{\partial \zeta_{1}}{\partial \rho}-\frac{\partial^{2}}{\partial \zeta^{2}} \frac{\partial \zeta_{1}}{\partial \rho}-\frac{1}{\rho^{2}} \frac{\partial \zeta_{1}}{\partial \rho} \\
& =\frac{2}{\rho}\left(-\frac{1}{\rho} \frac{\partial \zeta_{1}}{\partial \rho}-\frac{1}{\rho^{2}} \frac{\partial^{2} \zeta_{1}}{\partial \vartheta^{2}}\right) .
\end{aligned}
$$

Changing all signs and combining the second term in the 
left-hand side with the second term in the right-hand side, the equation reads

$$
\frac{1}{\rho} \frac{\partial}{\partial \rho} \rho \frac{\partial}{\partial \rho} \frac{\partial \zeta_{1}}{\partial \rho}+\frac{\partial}{\partial \rho} \frac{1}{\rho^{2}} \frac{\partial^{2} \zeta_{1}}{\partial \vartheta^{2}}+\frac{\partial^{2}}{\partial \zeta^{2}} \frac{\partial \zeta_{1}}{\partial \rho}+\frac{1}{\rho^{2}} \frac{\partial \zeta_{1}}{\partial \rho}=\frac{2}{\rho^{2}} \frac{\partial \zeta_{1}}{\partial \rho} .
$$

The first term in the left-hand side can be split as

$$
\begin{aligned}
\frac{1}{\rho} \frac{\partial}{\partial \rho}\left[\rho \frac{\partial}{\partial \rho} \frac{\partial \zeta_{1}}{\partial \rho}\right] & =\frac{1}{\rho} \frac{\partial}{\partial \rho}\left[\frac{\partial}{\partial \rho} \rho \frac{\partial \zeta_{1}}{\partial \rho}-\frac{\partial \zeta_{1}}{\partial \rho}\right] \\
& =\frac{\partial}{\partial \rho}\left[\frac{1}{\rho} \frac{\partial}{\partial \rho} \rho \frac{\partial \zeta_{1}}{\partial \rho}\right]+\frac{1}{\rho^{2}} \frac{\partial}{\partial \rho} \rho \frac{\partial \zeta_{1}}{\partial \rho}-\frac{1}{\rho} \frac{\partial^{2} \zeta_{1}}{\partial \rho^{2}} \\
& =\frac{\partial}{\partial \rho}\left[\frac{1}{\rho} \frac{\partial}{\partial \rho} \rho \frac{\partial \zeta_{1}}{\partial \rho}\right]+\frac{1}{\rho^{2}} \frac{\partial \zeta_{1}}{\partial \rho}
\end{aligned}
$$

so that finally the equation reads

$$
\frac{\partial}{\partial \rho}\left[\frac{1}{\rho} \frac{\partial}{\partial \rho} \rho \frac{\partial \zeta_{1}}{\partial \rho}+\frac{1}{\rho^{2}} \frac{\partial^{2} \zeta_{1}}{\partial \vartheta^{2}}+\frac{\partial^{2} \zeta_{1}}{\partial \zeta^{2}}\right]+\frac{2}{\rho^{2}} \frac{\partial \zeta_{1}}{\partial \rho}=\frac{2}{\rho^{2}} \frac{\partial \zeta_{1}}{\partial \rho} .
$$

This represents an identity since the sum in square brackets is $\nabla^{2} \zeta_{1}=0$, and the proof is therefore completed.

\section{APPENDIX B: TRANSFORMATION FROM FLUX TO DEVICE COORDINATES}

All calculations shown in this paper have been performed in flux coordinates. To plot the results in device coordinates a suitable transformation must be used.

"Cartesian flux coordinates" can be introduced starting from Eq. (21). Neglecting terms of order higher than $\epsilon^{1}$, one has

$$
X \equiv \rho \cos \vartheta \approx r \cos \theta+\epsilon\left[-\rho_{1} \cos \theta+r \vartheta_{1} \sin \theta\right]
$$

and

$$
Y \equiv \rho \sin \vartheta \approx r \sin \theta+\epsilon\left[-\rho_{1} \sin \theta-r \vartheta_{1} \sin \theta\right] .
$$

Substituting

$$
\begin{aligned}
& \rho_{1}=\left[\int_{\zeta_{0}}^{\zeta} \alpha(\zeta) d \zeta-\frac{3}{8} \alpha^{\prime}(\zeta) \rho^{2}\right] \cos \vartheta, \\
& \vartheta_{1}=\left[-\frac{1}{\rho} \int_{\zeta_{0}}^{\zeta} \alpha(\zeta) d \zeta+\frac{1}{8} \alpha^{\prime}(\zeta) \rho\right] \sin \vartheta,
\end{aligned}
$$

from Eq. (56) with $B_{1 x} / B_{*} \equiv \alpha$, one finally obtains

$$
\begin{aligned}
& X=x-\epsilon \int \alpha(z) d z+\epsilon \alpha^{\prime}(z)\left[\frac{3}{8} x^{2}+\frac{1}{8} y^{2}\right], \\
& Y=x+\epsilon \alpha^{\prime}(z)\left[\frac{1}{4} x y\right] .
\end{aligned}
$$

${ }^{1}$ J. H. Malmberg and C. F. Driscoll, Phys. Rev. Lett. 44, 654 (1980).

${ }^{2}$ D. H. E. Dubin and T. M. O’Neil, Phys. Plasmas 5, 1305 (1998).

${ }^{3}$ D. D. Ryutov and G. V. Stupakov, in Reviews of Plasma Physics, edited by B. B. Kadomtsev (Consultants Bureau, New York, 1987), Vol. 13, pp. 93-202.

${ }^{4}$ J. P. Freidberg, Ideal Magnetohydrodynamics (Plenum, New York, 1987).

${ }^{5}$ R. Keinigs, Phys. Fluids 24, 860 (1981).

${ }^{6}$ R. Keinigs, Phys. Fluids 27, 1427 (1984).

${ }^{7}$ E. P. Gilson and J. Fajans, in Non-Neutral Plasma Physics IV, edited by F. Anderegg, L. Schweikhard, and C. F. Driscoll (American Institute of Physics, New York, 2002), Vol. 606, p. 378.

${ }^{8}$ E. P. Gilson and J. Fajans, Phys. Rev. Lett. 90, 015001 (2003).

${ }^{9}$ G. W. Hart, Phys. Fluids B 3, 2987 (1991).

${ }^{10}$ R. Chu, J. S. Wurtele, J. Notte, A. J. Peurrung, and J. Fajans, Phys. Fluids B 5, 2378 (1993).

${ }^{11}$ K. Gomberoff, J. Fajans, A. Friedman, D. Grote, J.-L. Vay, and J. S. Wurtele, Phys. Plasmas 14, 102111 (2007).

${ }^{12}$ M. Amoretti, C. Amsler, G. Bonomi, A. Bouchta, P. Bowe, C. Carraro, C. L. Cesar, M. Charlton, M. J. T. Collier, M. Doser, V. Filippini, K. S. Fine, A. Fontana, M. C. Fujiwara, R. Funakoshi, P. Genova, J. S. Hangst, R. S. Hayano, M. H. Holzscheiter, L. V. Jørgensen, V. Lagomarsino, R. Landua, D. Lindelöf, E. Lodi Rizzini, M. Macrì, N. Madsen, G. Manuzio, M. Marchesotti, P. Montagna, H. Pruys, C. Regenfus, P. Riedler, J. Rochet, A. Rotondi, G. Rouleau, G. Testera, A. Variola, T. L. Watson, and D. P. van der Werf, Nature (London) 419, 456 (2002).

${ }^{13}$ G. Gabrielse, N. S. Bowden, P. Oxley, A. Speck, C. H. Storry, J. N. Tan, M. Wessels, D. Grzonka, W. Oelert, G. Schepers, T. Sefzick, J. Walz, H. Pittner, T. W. Hänsch, E. A. Hessels, and Atrap Collaboration, Phys. Rev. Lett. 89, 213401 (2002).

${ }^{14}$ I. Kotelnikov, M. Romé, and A. Kabantsev, Phys. Plasmas 13, 092108 (2006).

${ }^{15}$ T. S. Pedersen and A. H. Boozer, Phys. Rev. Lett. 88, 205002 (2002).

${ }^{16}$ K. Miyamoto, Fundamentals of Plasma Physics and Controlled Fusion (Iwanami, Tokyo, 1997).

${ }^{17}$ G. V. Stupakov, Fiz. Plazmy 5, 871 (1987); [Sov. J. Plasma Phys. 5, 486 (1979)].

${ }^{18}$ T. M. O'Neil, private communication (2005).

${ }^{19}$ K. S. Fine, Phys. Fluids B 4, 3981 (1992).

${ }^{20}$ R. J. Briggs, J. D. Daugherty, and R. Levy, Phys. Fluids 13, 421 (1970).

${ }^{21}$ A. V. Arefiev, I. A. Kotelnikov, M. Romé, and R. Pozzoli, Plasma Phys. Rep. 28, 141 (2002).

${ }^{22}$ L. Brillouin, Phys. Rev. 67, 260 (1945).

${ }^{23}$ I. Kotelnikov, M. Romé, and R. Pozzoli, Phys. Lett. A 372, 1445 (2008).

${ }^{24}$ M. D. Kruskal and R. M. Kulsrud, Phys. Fluids 1, 265 (1958).

${ }^{25}$ Performing the same calculations with the use of the coordinates $(\rho, \vartheta, \zeta)$ leads to the appearance of extra factors similar to $B_{*} \rho$ in intermediate formulas, which are avoided here for the sake of brevity.

${ }^{26}$ T. M. O'Neil, Comments Plasma Phys. Controlled Fusion 5, 213 (1980).

${ }^{27}$ I. Kotelnikov, R. Pozzoli, and M. Romé, Phys. Plasmas 7, 4396 (2000).

${ }^{28}$ M. Amoretti, G. Bettega, F. Cavaliere, M. Cavenago, F. De Luca, R. Pozzoli, and M. Romé, Rev. Sci. Instrum. 74, 3991 (2003).

${ }^{29}$ T. M. O'Neil and C. F. Driscoll, Phys. Fluids 22, 266 (1979).

${ }^{30}$ J. Fajans, Phys. Plasmas 10, 1209 (2003).

${ }^{31}$ Alternatively, one could combine the terms proportional to $\alpha^{\prime}$ in Eq. (96) into a single term, but this would give a function $\widetilde{\phi}_{1}^{(2)}=\phi_{1}^{(2)}-3 / 8 \phi_{1}^{(1)}$ that turns out to be more cumbersome than $\phi_{1}^{(2)}$.

${ }^{32}$ K. Gomberoff, J. Fajans, J. Wurtele, A. Friedman, D. P. Grote, and R. H. Cohen, Phys. Plasmas 14, 052107 (2007). 